\title{
THE IMPACT OF COMPETITION BETWEEN CANCER CELLS AND HEALTHY CELLS ON OPTIMAL DRUG DELIVERY
}

\author{
Heyrim $\mathrm{CHO}^{1, *}$ AND Doron Levy ${ }^{2,3, *}$
}

\begin{abstract}
Cell competition is recognized to be instrumental to the dynamics and structure of the tumor-host interface in invasive cancers. In mild competition scenarios, the healthy tissue and cancer cells can coexist. When the competition is aggressive, competitive cells, the so called super-competitors, expand by killing other cells. Novel chemotherapy drugs and molecularly targeted drugs are commonly administered as part of cancer therapy. Both types of drugs are susceptible to various mechanisms of drug resistance, obstructing or preventing a successful outcome. In this paper, we develop a cancer growth model that accounts for the competition between cancer cells and healthy cells. The model incorporates resistance to both chemotherapy and targeted drugs. In both cases, the level of drug resistance is assumed to be a continuous variable ranging from fully-sensitive to fully-resistant. Using our model we demonstrate that when the competition is moderate, therapies using both drugs are more effective compared with single drug therapies. However, when cancer cells are highly competitive, targeted drugs become more effective. The results of the study stress the importance of adjusting the therapy to the pre-treatment resistance levels. We conclude with a study of the spatiotemporal propagation of drug resistance in a competitive setting, verifying that the same conclusions hold in the spatially heterogeneous case.
\end{abstract}

Mathematics Subject Classification. 35Q92, 92B05.

Received December 5, 2018. Accepted October 3, 2019.

\section{INTRODUCTION}

Intra-tumor heterogeneity that results from both genetic and non-genetic mechanisms has been receiving increased attention in recent years $[29,37,53-55]$. Due to phenotypic and mutagenic diversity, cancer can be thought of as an ecosystem formed by coexisting populations expressing abnormal features and different cell types that are embedded in a heterogeneous habitat of normal tissue [39]. Accordingly, competition between tumor cells and healthy cells in the host tissue may play a key role in cancer growth [30,61]. Although the mechanisms are complex and are not fully understood, it is known that cells can discriminate their types via short-range interactions that quantify the relative expression levels of particular proteins. Accordingly, cell competition occurs in the process of identifying and eliminating the less fit cells. The fitness-induced process generally eliminates the defective cells, such as Minute gene mutated cells in D. melanogaster [62, 77]. However,

Keywords and phrases: Tumor growth, cell competition, drug resistance, chemotherapy, targeted drugs.

1 Department of Mathematics, University of California, Riverside, CA 92521, USA.

2 Department of Mathematics, University of Maryland, College Park, MD 20742, USA.

3 Center for Scientific Computation and Mathematical Modeling, University of Maryland, College Park, MD 20742, USA.

* Corresponding author: heyrimc@ucr.edu, dlevy@math.umd.edu

(C) The authors. Published by EDP Sciences, 2020 


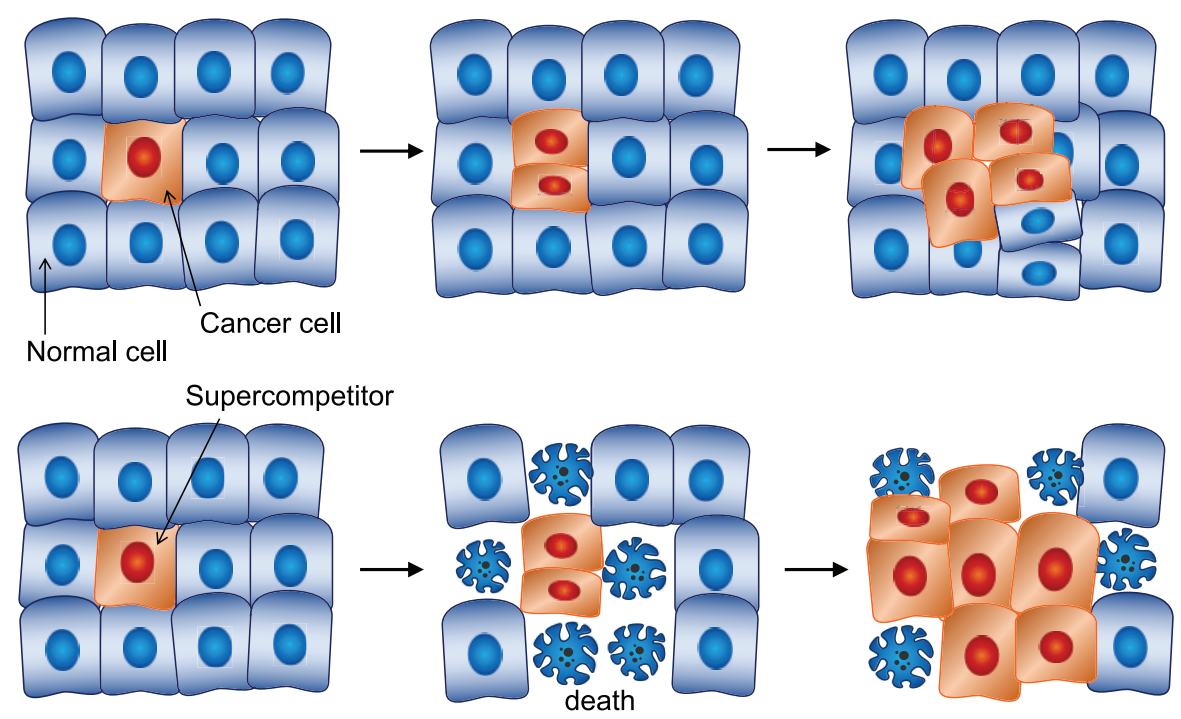

Figure 1. Competition between healthy cells and cancer cells. Often cancer cells and normal cells coexist and the expansion is restricted by spatial competition (top). However, some cancer cells, the so called super-competitors, expand by killing the healthy cells (bottom). Diagram adapted from [61].

certain types of cancer cells can signal the death of their surrounding tissue in a way that promotes their neoplastic transformation. After the "loser" cells disappear from the tissue, the "winner" cells not only survive but also proliferate to fill out the void created by the dying cells [19, 43, 61, 82]. Figure 1 illustrates cancer growth in two distinct competition scenarios.

To eliminate cancer cells and suppress their malignant growth, various treatments are available, including surgery, chemotherapy, immunotherapy, and radiotherapy. In particular, cytotoxic chemotherapy and molecular targeted approaches represent two modes of cancer treatment [56, 73]. Whereas chemotherapy uses highly potent chemicals to target dividing cells, targeted drugs act on specific molecular targets that are associated with cancer. In this paper, we are particularly interested in targeted drugs that have cytotoxic effects [23, 28, 42, 63, 72]. Although targeted drugs are often cytostatic, high dosages have cytotoxic effects. Examples include Erlotinib with certain non-small-cell lung cancer cell lines such as HCC827 [23], and gemtuzumab ozogamicin with myeloid leukemic cells. Novel therapies and drug substances are constantly being developed [22, 76].

For both chemotherapy and targeted therapy, drug resistance is the predominant factor limiting clinical success $[31,38,56,81]$. For instance, resistance to chemotherapy includes extrinsic mechanisms that prevent the drug from reaching its target in an active form due to short serum half-life or rapid clearance by the kidneys and liver [9, 78]. Intrinsic cellular mechanisms involve increased efflux or decreased uptake, enzymatic modification and inactivation of the drug, and alteration of drug targets within the cell [20,33,34]. Resistance to targeted drugs also relies on various mechanisms including cellular responses that maintain the signaling despite the effective targeting or signaling through alteration of downstream effectors, and cell survival pathways by disabling apoptosis $[10,88]$. Drug resistance involves genetic and epigenetic alternations that either exist prior to the treatment or acquired, often induced by the drugs [31, 81]. Clinical trials of combinations of cytotoxic and targeted drugs suggest that the complementing mechanisms can be used for developing effective therapies $[18,73]$.

Due to the complexity of the underlying mechanisms and the multifactorial pathways of tumor growth and drug resistance, various mathematical models have been developed to describe and investigate the emergence of cancer and its evolution. Modeling approaches include deterministic models using differential equations $[1,5,83]$ 
and stochastic models including branching processes and multiple mutations for studying multi-drug resistance and optimal control of drug scheduling $[46,47,58]$. In particular, tumor heterogeneity regarding drug resistance has been modeled as a phenotpe-structured integro-differential equation by considering a continuous phenotypic variable of resistance $[13,14,17,36,49,50]$. These modeling approaches have provided a framework for improving early detection, for quantifying intrinsic and acquired resistance cells, and for designing therapeutic protocols $[21,48,58,74,80]$.

On top of that, various mathematical models incorporate tumor heterogeneity and competition between distinct cell types [3, 51, 66, 87]. Recent models using ordinary differentiation equations (ODEs) focus on competition between distinct types of cancer cells that are either resistant or sensitive to a single drug [11, 66, 87]. While ODEs can model the overall size of the population, partial differential equations can model spatial heterogeneity in either the physical space or in the phenotypic space. Competition models using reaction-diffusion equations date back to [24] that describe the spatial distribution and temporal evolution of an invasive tumor, accounting for the density of the normal tissue and the neoplastic cancerous tissue. Considering phenotypic heterogeneity, [51] developed a model for the competition between healthy cells and tumor cells that depends on a continuous phenotypic variable of cytotoxic drug resistance level. References [68,69] further analyzed asymptotic behaviors of phenotype structured competition models of multiple population studying conditions for coexistence, trait selection, and optimal control of treatments. All aforementioned models consider drug resistance to a single drug or a single resistance trait.

To study the impact of cell competition and the heterogeneity in drug resistance, we develop a phenotypic structured model extending the model proposed in [51,69]. Our model consists of healthy cells and tumor cells having a state that depends on a continuous variable that represents the level of drug resistance. The model is aimed at designing effective combination therapies (see also $[13,14]$ ). We study two scenarios: (i) a mild competition that allows coexistence of distinct cells; and (ii) an aggressive competition that results with the elimination of one population (see Fig. 1). We examine the tumor response under a combination therapy of chemotherapy and targeted drug, addressed in the text as cytotoxic and targeted drug, respectively. We assume a continuous level of drug resistance to each drug. This distinguishes our work from most other competition models that only consider resistance to a single drug [3, 11, 51]. Our study implies that the optimal order between the drugs as well as the duration of therapy, depend on the competition parameter and on the ratio of preexisting resistance to each drug.

The paper is organized as follows. In Section 2, we introduce the competition model between cancer cells and healthy cells with a multi-dimensional resistance trait. We estimate the range of the competition rate that corresponds to the super-competitive scenario, where only one population can survive. In Section 3, we numerically study our model, focusing on cancer growth and on the emergence of resistance under different combination therapies. Therapies in which one drug is switched for a second drug are compared to single-drug therapies in Section 3.1, particularly when the competition rate is low. In Section 3.2, we study the effect of different models that characterize fitness in the resistance space and numerically compute the optimal switching time that minimizes the overall number of cancer cells in a given time interval. Alternating therapies and combination on-off therapies are compared in Section 3.3. The model is extended to space and the proposed therapies are studied in Section 4. Concluding remarks are provided in Section 5.

\section{A mathematical model FOR THE COMPETition BETWEen HeAlthy CELLS AND CANCER CELLS}

To model the competition between healthy cells and cancer cells and the emergence of resistance, we consider two populations: healthy cells $n_{h}(t, \theta)$ and cancer cells $n_{c}(t, \theta)$. Both populations describe the number of cells at time $t$ that have a resistance phenotype $\theta$. The variable $\theta=\left(\theta_{1}, \theta_{2}\right) \in[0,1]^{2}$ describes the level of drug resistance to cytotoxic drugs $\left(\theta_{1}\right)$ and to targeted drugs $\left(\theta_{2}\right)$. The value 0 corresponds to full sensitivity to the drug, and the value 1 corresponds to complete resistance. For example, the level of resistance to cytotoxic agents can be related to the expression level of a gene or a gene cluster that is linked to the cellular level of drug resistance and proliferation potential, such as MDR1, ALDH1, CD44 $[2,37,57]$. We model the competition of $n_{h}(t, \theta)$ and 
$n_{c}(t, \theta)$ as a reaction-diffusion system,

$$
\begin{aligned}
\partial_{t} n_{h}(t, \theta) & =\underbrace{\left[r_{h}(\theta)-d_{h}(\theta) \rho_{h}(t)-\mu_{h}(\theta) c_{1}(t)-\varphi_{h}(\theta) c_{2}(t)-\bar{a}_{h c}(\theta) \rho_{c}(t)\right]}_{G_{h}(t, \theta)} n_{h}+\nu_{h} \Delta_{\theta} n_{h}, \\
\partial_{t} n_{c}(t, \theta) & =\underbrace{\left[r_{c}(\theta)-d_{c}(\theta) \rho_{c}(t)-\mu_{c}(\theta) c_{1}(t)-\varphi_{c}(\theta) c_{2}(t)-\bar{a}_{c h}(\theta) \rho_{h}(t)\right]}_{G_{c}(t, \theta)} n_{c}+\nu_{c} \Delta_{\theta} n_{c},
\end{aligned}
$$

with Neumann boundary conditions with zero normal derivative. The reaction terms involve proliferation, apoptosis, and drug effect. The first reaction terms with $r_{h}(\theta) \geq 0$ and $r_{c}(\theta) \geq 0$ model the proliferation depending on the resistance level. We assume that the proliferation rates satisfy $\partial_{\theta_{i}} r_{h}(\theta) \leq 0$ and $\partial_{\theta_{i}} r_{c}(\theta) \leq 0$, corresponding to the assumption that resistant cells devote their resources to developing and maintaining the drug resistance mechanisms (see the experimental evidence in $[59,63,86]$ ).

The death terms involve the rate of apoptosis, $d_{h}(\theta) \geq 0$ and $d_{c}(\theta) \geq 0$. We consider a logistic growth model with $\rho_{h}(t)$ and $\rho_{c}(t)$ being the total numbers of normal cells and cancer cells, computed as

$$
\rho_{h}(t)=\int n_{h}(\theta, t) \mathrm{d} \theta, \quad \rho_{c}(t)=\int n_{c}(\theta, t) \mathrm{d} \theta,
$$

and $\rho(t)=\rho_{h}(t)+\rho_{c}(t)$. The carrying capacity of normal cells with phenotype $\theta$, is given by $r_{h}(\theta) / d_{h}(\theta)$. Key to the model are the competition terms: apoptosis due to competition occurs with rates $\bar{a}_{h c}$ and $\bar{a}_{c h}$ with respect to the size of the other cell population. This resembles the competitive Lotka-Volterra model (e.g., [24, 64]), and has been referred to as competition rate in competition models [66]. The competition rate is also related to phenotypic capacity. We consider $0 \leq \bar{a}_{h c} \leq d_{h}$ and $0 \leq \bar{a}_{c h} \leq d_{c}$. Positivity is assumed to account for spatial competition. The upper bounds account for interspecific competition that is typically lower than intra-specific competition.

The drug effects that represent the death of cancer cells due to the action of cytotoxic and targeted drugs are also included in the growth term. The time-dependent dosages are denoted by $c_{1}(t)$ for the cytotoxic drug and $c_{2}(t)$ for the targeted drug. The cancer cells respond to both the cytotoxic drug and the targeted drug with drug effect functions $\mu_{c}(\theta)$, and $\varphi_{c}(\theta)$, respectively. The healthy cells are affected by the cytotoxic drug with an effect function $\mu_{h}(\theta)$, and to a lesser degree by the targeted drug with $\varphi_{h}(\theta)$ compared to $\varphi_{c}(\theta)$. Chemotherapy uses highly potent chemicals that kill rapidly dividing cells, thus we take $\mu_{h}(\theta) \geq 0$ and $\mu_{c}(\theta) \geq 0$, and assume that the therapy is more effective with sensitive cells. On the other hand, targeted therapies selectively target these cancer-related genetic lesions. Hence, we let $\varphi_{c}(\theta) \geq 0$, and assume that the targeted drug affects healthy cells significantly less than cancer cells as $0 \leq \varphi_{h}(\theta) \ll \varphi_{c}(\theta)$. As the resistance level increases, the cells become more resilient to the drugs. This translates to the modeling assumption $\partial_{\theta_{i}} \mu_{h}(\theta) \leq 0, \partial_{\theta_{i}} \mu_{c}(\theta) \leq 0, \partial_{\theta_{i}} \varphi_{h}(\theta) \leq 0$, and $\partial_{\theta_{i}} \varphi_{c}(\theta) \leq 0[52,63]$.

The Laplacian operator $\Delta_{\theta}=\sum_{i=1}^{n} \partial^{2} / \partial \theta_{i}^{2}$ describes the instability in the resistance phenotypic space with rates $\nu_{h}$ and $\nu_{c}[17,49,50]$. In addition to genetic mutations, epimutations contribute to phenotypic instability: heritable changes in gene expression that do not alter the DNA $[8,26,27]$. Recent experiments demonstrated that such non-genetic instability and phenotypic variability allow cancer cells to reversibly transit between different phenotypic states $[12,67,75]$.

\subsection{Studying the competition parameter}

Recent studies suggest that cell competition is often critical in shaping cancer development [84, 85]. In particular, the fitness-sensing process during competition that usually eliminates defective cells, has a distinctive behavior in pre-cancerous lesions [61]. By acquiring a "super-fit" status, these super-competitors mutated cells can sense the surrounding wild-type cells as "less fit" and signal the death of surrounding tissue that in turn 
promotes their neoplastic transformation [30]. In our model, the competition parameters $\bar{a}_{h c}$ and $\bar{a}_{c h}$ describe the aggressiveness of the cells towards cells from the other populations.

To characterize competition scenarios, we simplify equations (2.1)-(2.2) by excluding the diffusion terms, and consider parameters as follows. We will revisit the diffusion terms in a later section. The proliferation rate and death rate are assumed to be constant. Cancer cells proliferate $A$ times faster than healthy cells, that is, $r_{h}=r$ and $r_{c}=A r$ with $A>1$. The apoptosis rates are taken as $d_{h}=d_{c}=d$. The cross-competition parameter is taken as $\bar{a}_{h c}=d a_{h c}$ and $\bar{a}_{c h}=d a_{c h}$, with $0 \leq a_{h c}, a_{c h} \leq 1$, so that $a_{h c}$ and $a_{c h}$ parameterize the relative magnitude of inter-specific competition to intra-specific competition. Moreover, we choose $\varphi_{h}=0$ to emphasize the distinctive targeted drug effect on the two cell populations that is $\varphi_{h} \ll \varphi_{c}$. We provide analytical results assuming that the model has a single trait, for instance, $n_{h}=\rho_{h} \delta_{\theta_{h}^{*}}$ and $n_{c}=\rho_{c} \delta_{\theta_{c}^{*}}$, and the simplified system can be rewritten as

$$
\begin{aligned}
& \dot{\rho}_{h}(t)=\left(r-d\left(\rho_{h}+a_{h c} \rho_{c}\right)-\mu_{h} c_{1}\right) \rho_{h} \\
& \dot{\rho}_{c}(t)=\left(A r-d\left(a_{c h} \rho_{h}+\rho_{c}\right)-\mu_{c} c_{1}-\varphi_{c} c_{2}\right) \rho_{c}
\end{aligned}
$$

with the coefficients evaluated at the single traits, for instance, $\mu_{h}=\mu_{h}\left(\theta_{h}^{*}\right)$. This is of value since phenotypestructured models may asymptotically converge to a Dirac-delta distribution at few dominating resistance traits without epimutation [68, 69], and in the limit of small mutation or epimutation with scale separation [51, 65]. Let us consider $\theta_{h}^{*}$ and $\theta_{c}^{*}$ as the traits that maximizes the growth rate of each equation.

We consider the following scenarios:

- No treatment. When no drug is applied $\left(c_{1}=c_{2}=0\right)$, we have $\theta_{h}^{*}=\theta_{c}^{*}=(0,0)$ since the most sensitive cells have the maximum growth rate. Then, a nontrivial equilibrium of $\rho_{h}>0$ and $\rho_{c}>0$ exists if $r-$ $d\left(\rho_{h}+a_{h c} \rho_{c}\right)=0$ and $A r-d\left(a_{c h} \rho_{h}+\rho_{c}\right)=0$ have strictly positive solutions. This reduces to a condition that the two populations can coexist when the competition rate satisfies $a_{h c}<r / A r<1 / a_{c h}$, where the latter inequality is satisfied since $a_{c h} \leq 1$. Otherwise, when the competition rate is $a_{h c}>1 / A$, one of the cell populations must become extinct, either $\rho_{h}>0, \rho_{c}=0$ or $\rho_{h}=0, \rho_{c}>0$. We consider this case as the super-competitive scenario. Since $A$ represents the ratio of over-proliferation of cancer cells compared to normal cells, for larger values of $A$, it is likely to be super-competitive for a larger range of the competition rate $a_{h c}$.

- Treatment. The condition for coexistence changes when the drug is applied. For the cytotoxic drug, the condition for coexistence amounts to $a_{h c}<\left(r-\mu_{h} c_{1}\right) /\left(A r-\mu_{c} c_{1}\right)$, assuming that with dosage $c_{1}$, there exist cells with trait $\theta^{*}$ that achieve positive growth rates. If the growth rate is negative for all cells, the population will go extinct, and it is meaningless to discuss coexistence. We note that the condition of cytotoxic treatment is identical to the no treatment case if the drug effect rate of healthy cells versus cancer cells is proportional to the proliferation rate, that is, if $\mu_{c}=A \mu_{h}$. However, under targeted therapy, coexistence boils down to $a_{h c}<r /\left(A r-A \varphi_{c} c_{2}\right)$ so that the cells are likely to coexist for smaller values of $A$. For weak dosages such that $c_{1}<\min \left(r / \mu_{h}, A r / \mu_{c}\right)$ or $c_{2}<r / \varphi_{c}$, it is apparent that the coexistence condition may differ from the no treatment case, except when $\mu_{c}=A \mu_{h}$. However, when the drug dosages are sufficiently high, only the highly resistant cells, $\theta_{1}^{*} \approx 1$ and $\theta_{2}^{*} \approx 1$, can survive, and with our assumption that the drug effects of resistant cells are close to zero, for example, $\mu_{c}\left(\theta_{1}^{*}=1\right) \approx 0$, the coexistence condition becomes similar to the no treatment case as $a_{h c}<1 / A$.

Figure 2 shows healthy cells, cancer cells, and combined counts, up to $t=100$, for $a_{h c}=0.0,0.2, \ldots, 1.0$ and $a_{c h}=a_{h c} / 2$. We consider $r_{h}(\theta)=r=1.5$ and $r_{c}(\theta)=2 r=3.0$, which corresponds to the relative proliferation $A=2$. Healthy cells and cancer cells coexist when $a_{h c}<1 / 2$, but when $a_{h c}>1 / 2$, the cancer cells overtakes the population and the healthy cells are eliminated. This condition is demonstrated again in Figure $3 \mathrm{a}$. The relative numbers of cells $\rho_{h}(t) / \rho(t)$ and $\rho_{c}(t) / \rho(t)$ at $t=50$ are plotted for different values of $A$ and $a_{h c}$. The coexistence threshold $1 / A$ is apparent in the results. We remark that other values of $a_{c h}$ in the range of $0 \leq a_{c h} \leq a_{h c}$ show similar results. 

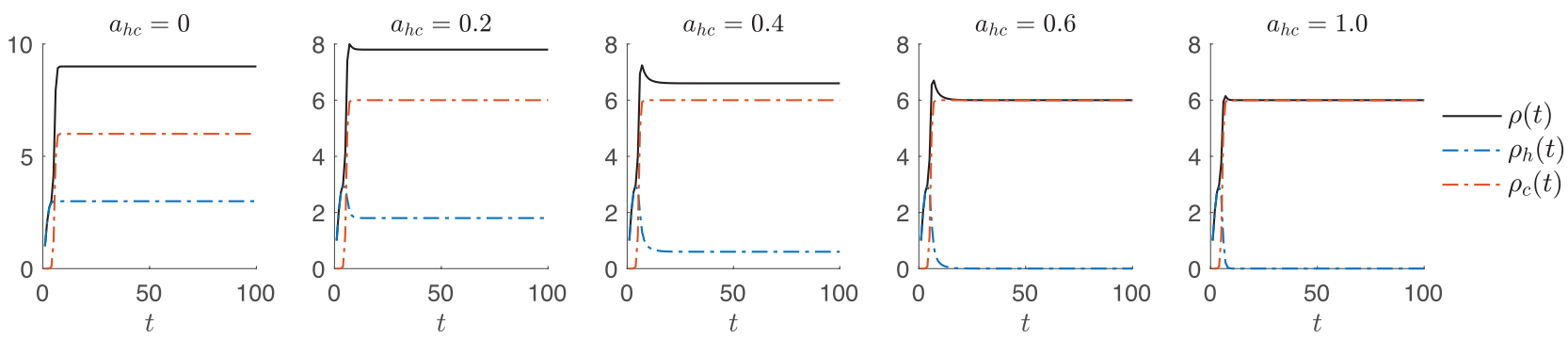

Figure 2. Comparison of the dynamics of the total number of cells $\rho(t)=\rho_{h}(t)+\rho_{c}(t)$ for different values of the competition parameter $a_{h c}=0,0.2, \ldots, 1$. The cancer cells proliferation factor is $A=2$ and the results are shown up to $t=40$. Healthy cells and cancer cells coexist when $a_{h c} \leq 1 / A=0.5$, while the cancer cells aggressively overtakes the population when $a_{h c}>0.5$. The latter case corresponds to the super-competitive model.
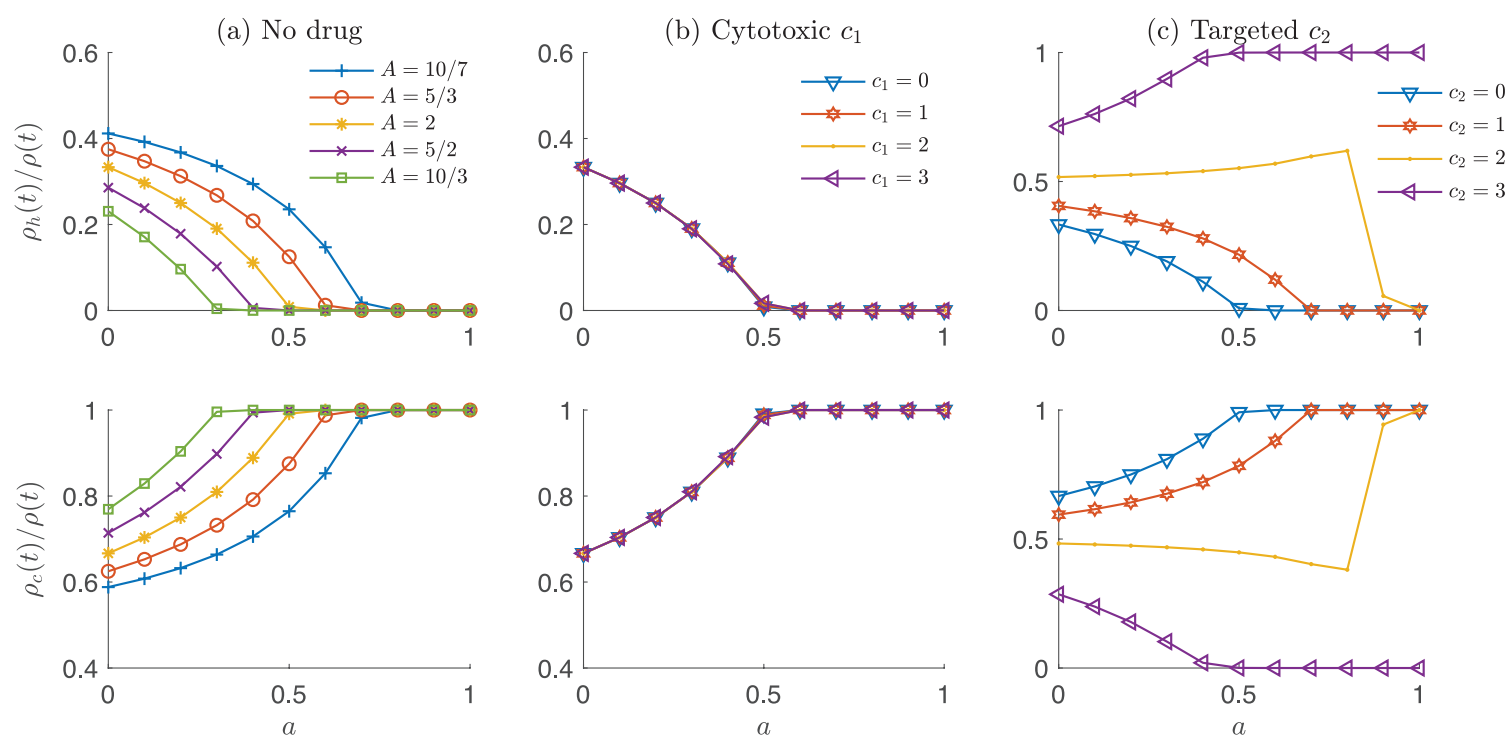

FiguRE 3 . The relative size of healthy cells $\rho_{h}(t) / \rho(t)$ (left) and cancer cells $\rho_{c}(t) / \rho(t)$ (right) at $t=50$ depending on the competition parameter $a_{h c}=a_{c h}=a$. (a) Without the drug treatment, cells coexist when the competition parameter is $a_{h c} \leq 1 / A$. The second and third rows show the results for a fixed $A=2$. (b) The competition trend does not change when a cytotoxic drug is applied regardless of the dosage. (c) The targeted drug has a significant impact on the dynamics of coexistence as it only affects the cancer cells.

The results shown in Figure 3b,c are computed with $A=2$ and agree with the theoretical thresholds. While the cytotoxic drug does not change the range of $a_{h c}$ that corresponds to coexistence, the targeted drug increases the range of coexistence for weak dosages. The threshold of the competition parameter is summarized in Table 1.

\subsection{Effectiveness of targeted drugs regarding competition}

In this section, we study how the competition rate affects the efficacy of treatments. We denote the trait of dominating population of cancer cells at time $t$ as, $\theta^{*}=\theta^{*}(t)=\arg \max _{\theta} n_{c}(t, \theta)$, and examine the growth term 
TABLE 1 . The range of the competition parameter within $0 \leq a_{h c} \leq 1$ such that the competition model becomes aggressively competitive, not allowing for coexistence. The competition is likely to be aggressive when the over-proliferation ratio of the cancer cells $A$ is large. We note that the parameter range of the cytotoxic drug reduces to $a_{h c}>1 / A$ for any dosage if $\mu_{c}=A \mu_{h}$.

\begin{tabular}{lll}
\hline \multirow{2}{*}{ No treatment } & \multicolumn{2}{c}{ Treatment } \\
\cline { 2 - 3 }$a_{h c}>\frac{1}{A}$ & $a_{h c}>\frac{r-\mu_{h} c_{1}}{A r-\mu_{c} c_{1}}$ & $a_{h c}>\frac{r}{A r-A \varphi_{c} c_{2}}$ \\
\hline
\end{tabular}

of cancer cells. The instantaneous growth rate of cancer cells at time $t$ becomes

$$
G_{c}\left(t, \theta^{*}\right)=r_{c}\left(\theta^{*}\right)-d_{c}\left(\theta^{*}\right)\left(a_{c h} \rho_{h}(t)+\rho_{c}(t)\right)-\mu_{c}\left(\theta_{1}^{*}\right) c_{1}-\varphi_{c}\left(\theta_{2}^{*}\right) c_{2},
$$

where $\theta^{*}=\left(\theta_{1}^{*}, \theta_{2}^{*}\right)$. We focus on the second and fourth terms on the right-hand side of equation (2.4). Using a single drug treatment with cytotoxic drug, we can eliminate the dominating cancer cells with dosages that satisfy $G_{c}\left(t, \theta^{*}\right)<0$, i.e.,

$$
\frac{r_{c}\left(\theta^{*}\right)-d_{c}\left(\theta^{*}\right)\left(a_{c h} \rho_{h}(t)+\rho_{c}(t)\right)}{\mu_{c}\left(\theta_{1}^{*}\right)}<c_{1} .
$$

When the sensitive cells are dominant so that $\theta_{1}^{*} \approx 0$, the cytotoxic drug dosage that is necessary in order to lower the number of cancer cells is expected to be in a reasonable range within the limit of the maximum tolerated dose. However, knowing that $\mu_{c}$ is a decreasing function, as the dominating resistance trait $\theta_{1}^{*}$ increases, the left-hand side may reach the maximum tolerated dose, which will require $c_{1}$ to be beyond the maximal limit in order to shrink the cancer cell population.

Eventually, when $\theta_{1}^{*} \approx 1$, the cytotoxic drug effect term $\mu_{c}\left(\theta_{1}^{*}\right) c_{1}$ becomes negligible, that is, $\mu_{c}\left(\theta_{1}^{*}\right) \approx 0$, even with dosages higher than the maximum tolerated dose. Although the growth rate of the cancer cells $G_{c}\left(t, \theta^{*}\right)$ can no longer be reduced by the cytotoxic drug effect $\mu_{c}\left(\theta_{1}^{*}\right) c_{1}$, we realize that it can still be suppressed by the competition term $d_{c}\left(\theta^{*}\right) a_{c h} \rho_{h}(t)$. Thus, when the dominating cancer population cannot be reduced by a sufficiently large cytotoxic drug dosage $c_{1}$, for instance, $r_{c}\left(\theta^{*}\right)-\mu_{c}\left(\theta_{1}^{*}\right) c_{1}>0$, reducing the growth rate through the competition term involving the healthy cells can be more effective. In this case, targeted therapy is preferable since $\varphi_{h} \ll \varphi_{c}$ so that it can retain the size of $\rho_{h}(t)$, and the cancer cells can be suppressed by the competition term $d_{c}\left(\theta^{*}\right) a_{c h} \rho_{h}(t)$. The effect of this term becomes more substantial for larger values of the competition parameter $a_{c h}$.

In short, the targeted drug can be particularly effective when the competition parameter $a_{c h}$ is large, and when chemotherapy becomes ineffective due to resistance. We verify the effectiveness of targeted drugs in the highly competitive case in the following sections.

\section{Combining CYTOTOXIC AND TARGETED DRUGS}

We study the effect of different combination therapies with cytotoxic and targeted drugs regarding competition. We simplify the parameters by considering $a_{h c}=a_{c h}=a$, which takes into account of the super-competitors and also a highly competitive nature of fighting for space. We comment that taking an identical values for $a_{h c}$ and $a_{c h}$ in case of super-competitors is not biologically justified, and we will comment later in this section about distinct values of $a_{h c}$ and $a_{c h}$. We again note that $\bar{a}_{h c}=d_{h} a_{h c}$ and $\bar{a}_{c h}=d_{c} a_{c h}$ in equations (2.1)-(2.2). The resistance trait becomes $\theta=\left(\theta_{1}, \theta_{2}\right) \in[0,1]^{2}$, where $\theta_{1}$ and $\theta_{2}$ represents resistance to cytotoxic and targeted 
(a)

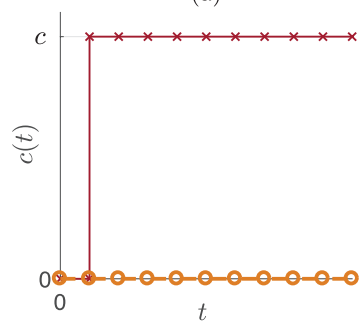

(b)

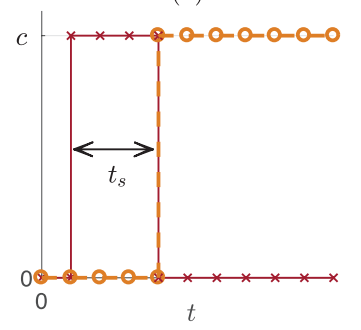

(c)

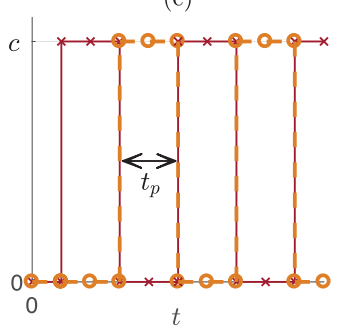

(d)

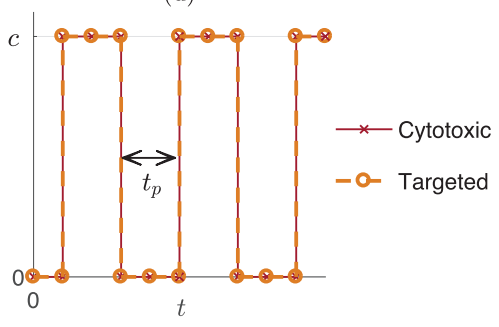

Figure 4. Drug scheduling considered in our simulations: (a) single cytotoxic drug therapy initiated at $t_{c}$; (b) drug switching therapy such that the drug is switched once after $t_{s} ;(\mathrm{c})$ alternating therapy with period $t_{p}$; and (d) on-off combination therapy with period $t_{p}$. We also test schedules of (a-c) initiated with targeted drugs.

drug, respectively. In particular, we consider the model functions as in [51],

$$
\begin{aligned}
& r_{h}(\theta)=\frac{\eta_{h}}{\prod_{i=1}^{2}\left(1+\theta_{i}^{2}\right)}, \quad r_{c}(\theta)=\frac{\eta_{c}}{\prod_{i=1}^{2}\left(1+\theta_{i}^{2}\right)}, \\
& d_{h}(\theta)=\frac{d}{\prod_{i=1}^{2}\left(1-0.1 \theta_{i}\right)}, \quad d_{c}(\theta)=\frac{d}{\prod_{i=1}^{2}\left(1-0.3 \theta_{i}\right)},
\end{aligned}
$$

where $\eta_{h}=1.5$ and $\eta_{c}=3$ are the maximum proliferation rates of healthy and cancer cells, respectively, and $d=0.5$ is the apoptosis rate. The relative proliferation of cancer cells over healthy cells is taken as $A=2$, similarly to the previous section so that $a=1 / A=0.5$ will distinguish the two competitive cases. The apoptosis terms are taken as in [51] in order to mimic the fact that mutations conferring resistance to therapies may also provide cells with stronger competitive abilities. The drug effect functions are taken as

$$
\begin{array}{ll}
\mu_{h}(\theta)=0.4\left(1-\theta_{1}\right), & \mu_{c}(\theta)=0.8\left(1-\theta_{1}\right), \\
\varphi_{h}(\theta)=0, & \varphi_{c}(\theta)=0.8\left(1-\theta_{2}\right) .
\end{array}
$$

All model functions satisfy the positivity and slope assumptions from Section 2 . The drug schedules we consider are shown in Figure 4. We consider four different therapies:

(a) a single cytotoxic drug therapy initiated at $t_{c}$, that is, $c_{i}(t)=c_{i} \mathbf{1}_{t_{c} \leq t}$,

(b) a switching therapy such that the drug is switched once after $t_{s}, c_{i}(t)=c_{i} \mathbf{1}_{t_{c} \leq t \leq t_{c}+t_{s}}$ and $c_{j}(t)=$ $c_{j} \mathbf{1}_{t_{c}+t_{s} \leq t}$

(c) an alternating therapy with period $t_{p}, c_{i}(t)=c_{i} \mathbf{1}_{(2 n) t_{p} \leq t-t_{c} \leq(2 n+1) t_{p}}$ and $c_{j}(t)=c_{j} \mathbf{1}_{(2 n-1) t_{p} \leq t-t_{c} \leq(2 n) t_{p}}$,

(d) combination on-off therapy with period $t_{p}, \quad c_{i}(t)=c_{i} \mathbf{1}_{(2 n) t_{p} \leq t-t_{c} \leq(2 n+1) t_{p}}$ and $c_{j}(t)=$ $c_{j} \mathbf{1}_{(2 n) t_{p} \leq t-t_{c} \leq(2 n+1) t_{p}}$.

The purpose of investigating different drug schedules is to delay the relapse as much as possible. A relapse is defined as the tumor growing back to a size that is comparable to its size prior to treatment. Since the model includes cancer cells that are resistant to both drugs, a relapse eventually occurs. Our goal is thus to delay the relapse as much as possible. This goal is consistent with the experiments reporting a delayed relapse followed by an eventual relapse that is obtained by considering combination therapies of chemotherapy and targeted drugs [32].

The initial cell populations are set as

$$
\begin{array}{r}
n_{h}(0, \theta)=\frac{1-w}{C_{0}} \exp \left[-\frac{\left(\theta_{1}-\bar{\theta}_{1}\right)^{2}}{\epsilon}-\frac{\left(\theta_{2}-\bar{\theta}_{2}\right)^{2}}{\epsilon}\right], \\
n_{c}(0, \theta)=\frac{w}{C_{0}} \exp \left[-\frac{\left(\theta_{1}-\bar{\theta}_{1}\right)^{2}}{\epsilon}-\frac{\left(\theta_{2}-\bar{\theta}_{2}\right)^{2}}{\epsilon}\right],
\end{array}
$$




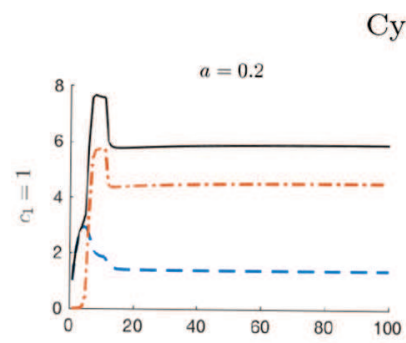

Cytotoxic
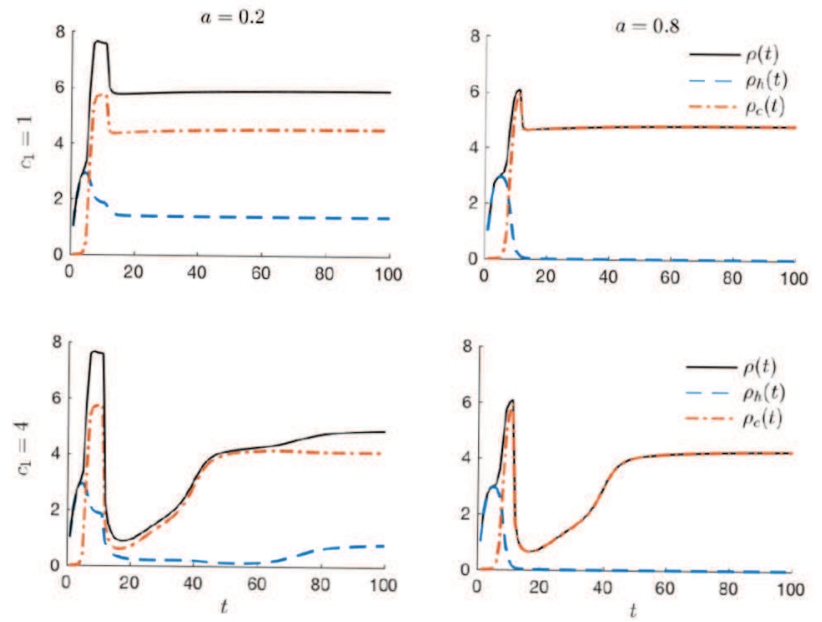

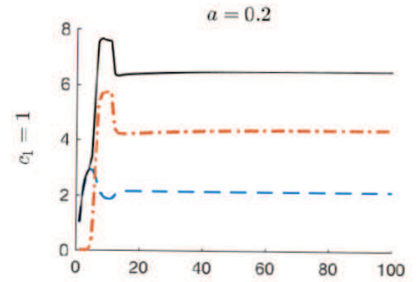

Targeted
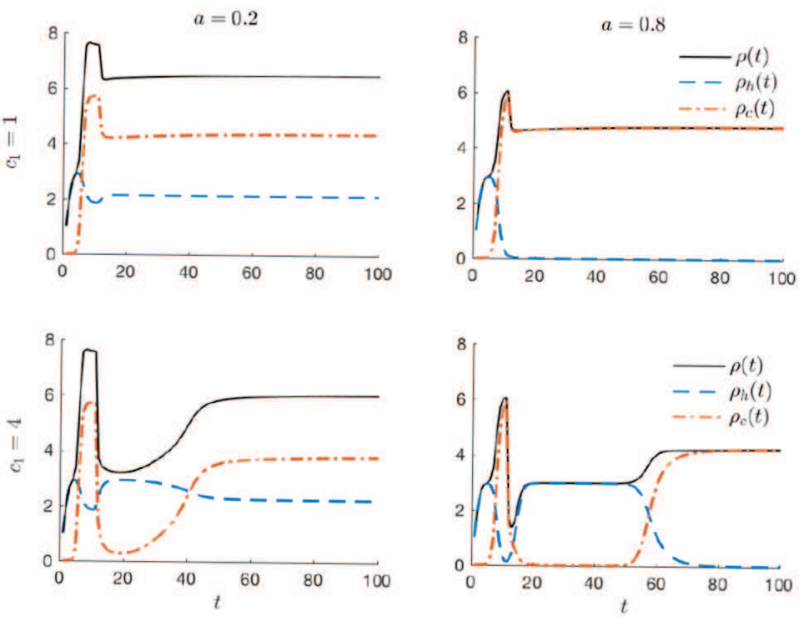

Figure 5. Comparison of the total number of cells $\rho(t)=\rho_{h}(t)+\rho_{c}(t)$ when using cytotoxic and targeted therapy with different values of the competition parameter $a=0.2$ and 0.8 . The drug is administered at $t_{c}=10$ with dosages $c_{i}=1$ and 4 . When $c_{1}=4$, administering a cytotoxic drug results with a relapse regardless of the values of $a$. The timing of the relapse is delayed in the case $a=0.8$ when administering a high dosage $c_{2}=4$ of the targeted drug.

where $\theta=\left(\theta_{1}, \theta_{2}\right) \in[0,1]^{2}$. Here, the mean resistance phenotype is centered at $\bar{\theta}_{1}$ and at $\bar{\theta}_{2}$ for the cytotoxic drug and the targeted drug, respectively. For a population without prior treatment, we typically assume $\bar{\theta}_{1}=0$ and $\bar{\theta}_{2}=0$. In addition, $w$ represents the initial proportion of the cancer cells in the tissue, and $\epsilon$ controls the variance of the preexisting resistance. $C_{0}$ is a normalizing constant chosen so that $\rho(0)=1$. For the numerical simulation, we consider a fourth-order finite difference method for the phenotypic space with 100 grid points and the fourth-order Runge-Kutta method with time step $10^{-3}$ for time integration.

\subsection{Single drug and drug switching therapy using cytotoxic and targeted drugs}

We first examine the outcome of a single drug therapy for different values of the competition parameter $a$, using either a cytotoxic or a targeted drug. We simulate the model without the diffusion term $\left(\nu_{h}=\nu_{c}=0\right)$. For the initial condition, we set $\bar{\theta}_{1}=\bar{\theta}_{2}=0, w=10^{-5}$, and $\epsilon=0.05$ [4, 41]. This initial condition corresponds to preexistence of $O\left(10^{-5} \%\right)$ resistant cells [40]. The results shown in Figure 5 confirm that the competition parameter $a$ determines the outcome: either coexistence or aggressive competition. This is the case with a low dosage $c_{i}=1$ as well as with a higher dosage $c_{i}=4$. When $a=0.2$, healthy cells and cancer cells are both present throughout the treatment, but not when $a=0.8$. This result holds for fixed $a_{h c}=0.2$ and $a_{h c}=0.8$, while varying the values of $a_{c h}$. In particular, under a high dosage of the cytotoxic drug, $c_{1}=4$, the relapsed cancer cells overtake the population. In the case of a targeted drug, the healthy cells suppress the cancer cells for some time, so that the relapse is delayed. This does not prevent an eventual relapse. The results are consistent with the observations of [79] that showed that when the APC mutant clones in Drosophila midgut reach a certain size, they induce the apoptotic death of the surrounding wild-type cells. From this simulation, we observe that the targeted drug is partially effective in the competitive scenario, $a=0.8$.

Figures 6 and 7 show the effect of increasing the dosages in the range $2 \leq c_{i} \leq 6$ for the single drug therapy case, comparing cytotoxic and targeted drugs. Here, we assume less preexisting resistance of $O\left(10^{-8} \%\right)$ resistant cells by setting $\epsilon=0.02$, and such assumption yields delayed relapse compared to $\epsilon=0.05$. In Figure 6 , acute relapse is observed under cytotoxic drug with high dosages $c_{i}=5$, 6 , where the number of cancer cells rapidly increases at later times $(t>50)$, compared with moderate dosages $\left(c_{i}=2,3\right)$. This is the case for both 

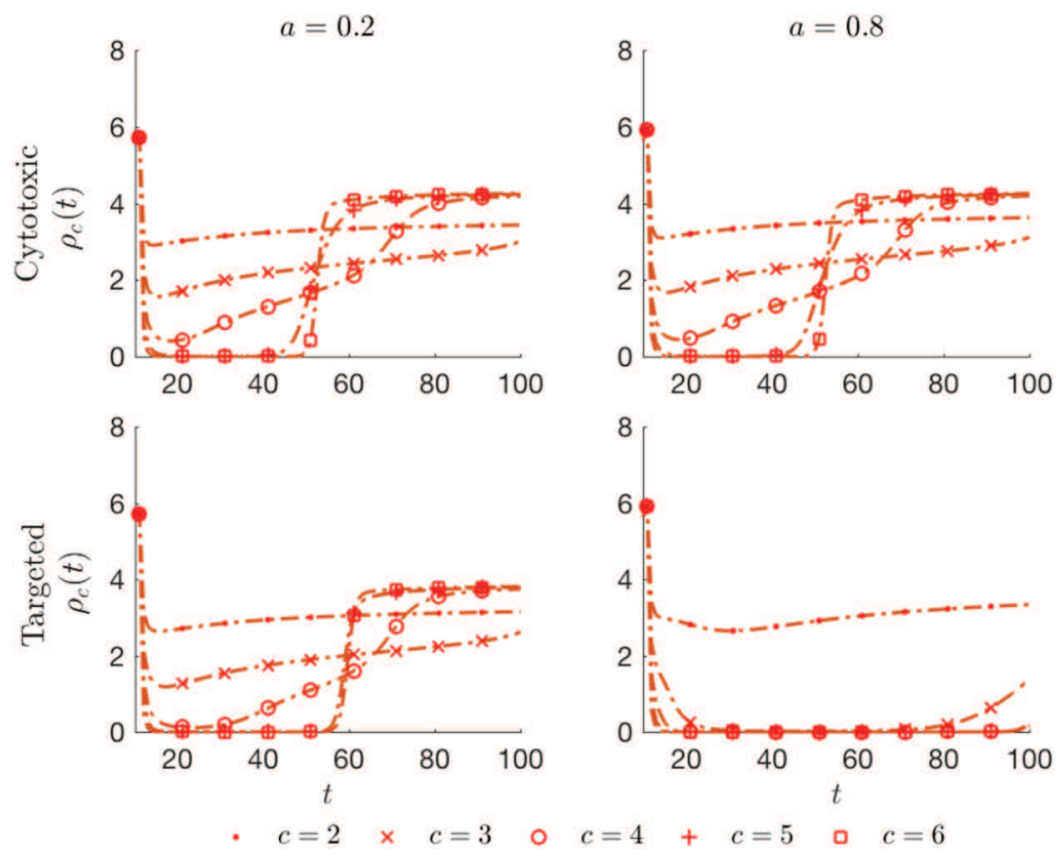

FIgURE 6. Comparison of the total number of cancer cells $\rho_{c}(t)$ for an increased drug dosage $2 \leq$ $c \leq 6$. The results compare cytotoxic therapy $c_{1}=c($ top $)$ and targeted therapy $c_{2}=c$ (bottom) for the competition parameters $a=0.2$ (left) and $a=0.8$ (right). We observe that in general, high cytotoxic drug dosages $\left(c_{i}=5,6\right)$ result with a delayed, yet stronger relapse compared with moderate dosages $\left(c_{i}=2,3\right)$. In contrast, targeted therapy results with a substantial delay in the relapse time in the highly competitive case $a=0.8$.
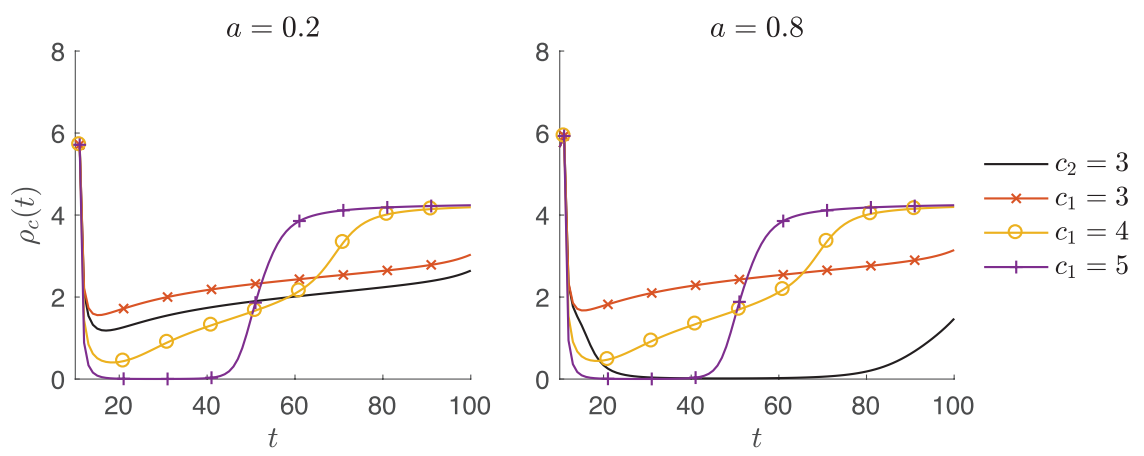

Figure 7. Comparison of the total number of cancer cells, $\rho_{c}(t)$, using cytotoxic and targeted therapy. The drug effect function of the cytotoxic drug is scaled such that it ranges from $100 \%$ to $166 \%$ of the drug effect function of the targeted drug. The cytotoxic drug with a higher cytotoxic effect reduces the cancer cells more effectively than the targeted drug until a certain time, especially when $a=0.2$. However, in the more competitive case, $a=0.8$, the targeted drug quickly becomes more effective.

competitive scenarios $a=0.2$ and 0.8. However, under targeted drugs, the relapse is worse when $a=0.2$, but not when the cells are highly competitive $(a=0.8)$. The targeted drugs eliminate only the cancer cells which helps the healthy tissue maintain its dominance and suppress the growth of cancer. In contrast, the cytotoxic drug, provides an advantage to the highly proliferative cancer cells, allowing them to fill in the void. 
We compare the results when the rate of apoptosis due to the cytotoxic drug is larger than the rate induced by the targeted drug, hoping that this will provides insights about improved drug scheduling. Figure 7 shows the results where the drug effect function of the cytotoxic drug amplifies by up to 1.66 times the effect of the targeted drugs. The cytotoxic drug with a stronger effect function is more efficient in killing the cancer cells, until a certain time point where the resistant cells cause a relapse. However, when $a=0.8$, stronger apoptosis of the cytotoxic drug holds for a very short period of time, so that the targeted drug has a significant advantage over the cytotoxic drug. This suggests a possibility of designing a more effective combination drug scheduling relative to a single drug therapy that shrinks the cancer cell population, particularly when the competition is less aggressive $(a<1 / A)$. It is likely that the targeted drug therapy may be more effective in highly competitive cases $(a>1 / A)$.

To demonstrate the effectiveness of combination therapies, we compare the number of cells $\rho(t)$ and $\rho_{c}(t)$ using a single drug therapy, to a combination therapy switching either from a cytotoxic drug to a targeted drug or the other way around. We comment that often the first-line therapy is replaced by other drugs once it becomes ineffective $[6,45,60]$. Figure 8 presents the results where the first drug is applied at $t=10$, and switched to the second drug at $t=20$. We set the values of the cytotoxic drug dosage, $c_{1}$, and the targeted drug dosage, $c_{2}$, to either 4 or 6 . We observe that switching the drug delays the relapse until the final simulation time $t=80$, in particular with the higher drug dosage, $c_{i}=6$. While a single drug therapy eventually results in a relapse due to the resistant population, we verify that switching the drug helps in delaying the relapse. This conclusion depends on the level of competition. With a moderate dosage $c_{i}=4$, switching from a cytotoxic drug to a targeted drug is effective when $a=0.2$, but not when $a=0.8$, where targeted drugs are advantageous.

We further aim to compute the optimal switching time. Figures 9 and 10 show the total number of cancer cells, $\rho_{c}(t)$, for the low-competition $(a=0.2)$ and the highly competitive $(a=0.8)$ cases. The drug is switched at different times after the initial drug is applied at $t_{c}=10$. We fix the dosage as $c_{i}=4$. The case when the therapy is initiated with a cytotoxic drug is shown in Figure 9. We observe that the cancer cell population remains low when the targeted drug is applied after the cytotoxic drug has eliminated sufficiently many cells that were resistant to the targeted drug. The second row and the right column show the relative size of the total cancer cell population compared to a single drug therapy, that is, $\sigma_{c} \doteq \int_{0}^{80} \rho_{c}(t) \mathrm{d} t / \int_{0}^{80} \rho_{c}^{*}(t) \mathrm{d} t$, where $\rho_{c}^{*}(t)$ is the number of cancer cells subject to a single drug treatment, in this case a cytotoxic drug. We observe that when $a=0.2$, there exists an optimal switching time. The relative cancer size is minimized to $20 \%$ when the therapy is switched at $t_{c}+20$. Figure 10 shows the opposite case where the drug is switched from targeted to cytotoxic. Similarly, one can benefit from switching the drug when $a=0.2$. In this case, the optimal switching time is approximately $t_{c}+30$. When the competition is mild, the optimal switching time is approximately after the time when the cancer grows back due to resistance. This holds for different initial conditions, in particular for an initial cancer population $10^{-6} \leq w \leq 10^{-4}$ and preexisting resistance $0.02 \leq \epsilon \leq 0.04$. When $a=0.8$, a therapy using only the targeted drug with a dosage of $c_{2}=4$ is more effective than an alternating therapy. As a result, rapidly switching the drug from targeted to cytotoxic yields significantly worse results, while for the switching from cytotoxic to targeted drug - the sooner the better. This holds unless there is a relatively large preexisting resistant population to the targeted drug $(\epsilon \geq 0.04)$. In this case, the initial application of a cytotoxic drug becomes effective in reducing preexisting resistant cells, although the optimal switching time does not exceed $t_{c}+8$. We remark that for different values of $a_{h c}$ and $a_{c h}$, the effectiveness of the targeted drug and the trend of optimal switching time is similar to Figures 9 and 10 when $a_{c h}$ is either 0.2 or 0.8 and the values of $a_{h c}$ vary.

\subsection{Linear versus nonlinear models of drug resistance fitness}

In this section, we study the implications of considering different models that characterize fitness in the resistance space on the treatment scheduling. Generally, we will observe variations in the mean resistance level as a function of the drug dosage. In a previous work we demonstrated that different continuum models of proliferation and drug effect functions yield distinctive dynamics in the drug resistance space [15]. The dynamics of continuum-resistance models can be similar to the dynamics of models that are based on discrete levels of 

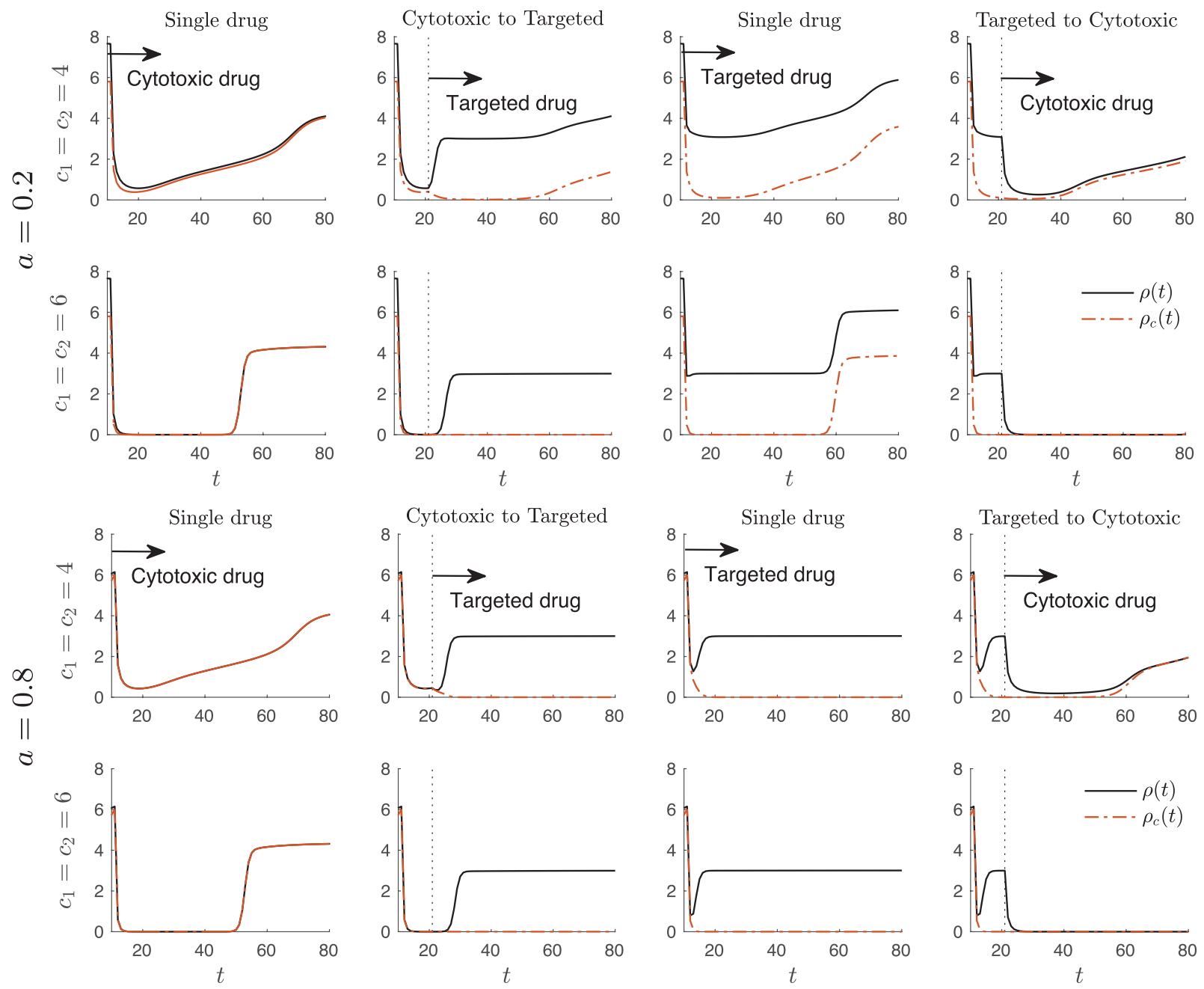

Figure 8. Comparison of the total number of cells $\rho(t)$ and cancer cells $\rho_{c}(t)$ using either a single drug therapy or a combination therapy, switching the drug once. The initial drug is applied at $t=10$ and switched to the second drug at $t=20$, that is, $t_{s}=10$. The top 2 rows correspond to $a=0.2$. Compared to the single drug therapies, switching the drug in either way delays the relapse. The bottom 2 rows are the highly competitive case when $a=0.8$.

resistance. It can also be significantly different. In linear models of proliferation $r_{h}(\theta)$ and $r_{c}(\theta)$ and drug effect $\mu_{h}(\theta)$ and $\mu_{c}(\theta)$ in equations (2.1)-(2.2), the typical outcome is that cells end up concentrating either in the most sensitive or in the most resistant trait. Such dynamics is essentially similar to considering a model with two resistance states: fully resistant and fully sensitive. Differences between the continuum and discrete models are observed with non-linear proliferation and drug effect functions. Here, we study how our model depends on the choice of continuum models by considering two functions: (i) a quadratic model that is concave down for proliferation and concave up for drug effect that allows intermediate resistance level,

$$
r_{c}(\theta)=\left(\eta_{c}-\frac{\eta_{c}}{4}\right) \prod_{i=1}^{2}\left(1-\theta_{i}^{2}\right)+\frac{\eta_{c}}{4}, \quad \mu_{c}(\theta)=0.8\left(\theta_{1}-1\right)^{2}, \quad \varphi_{c}(\theta)=0.8\left(\theta_{2}-1\right)^{2},
$$



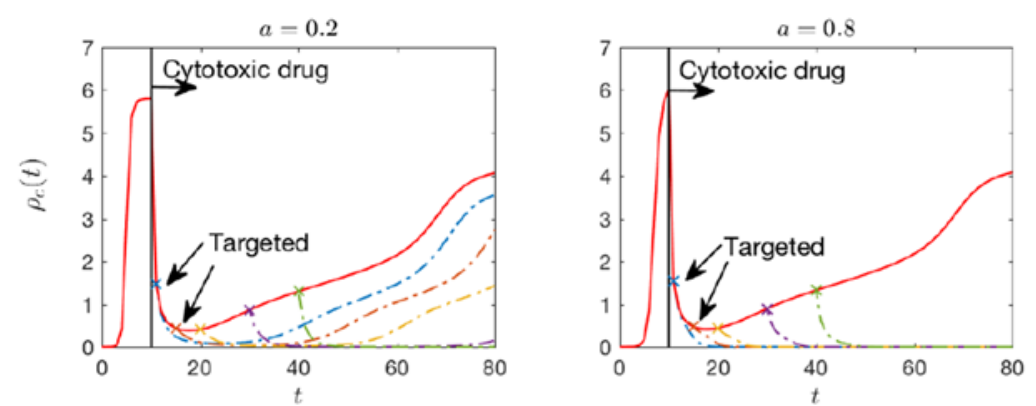

Cytotoxic $t_{c}=10-*$. Targeted with $t_{s}=1-*-\cdot t_{s}=5-*-\cdot t_{s}=10-* * \cdot t_{s}=20-*-\cdot t_{s}=30$
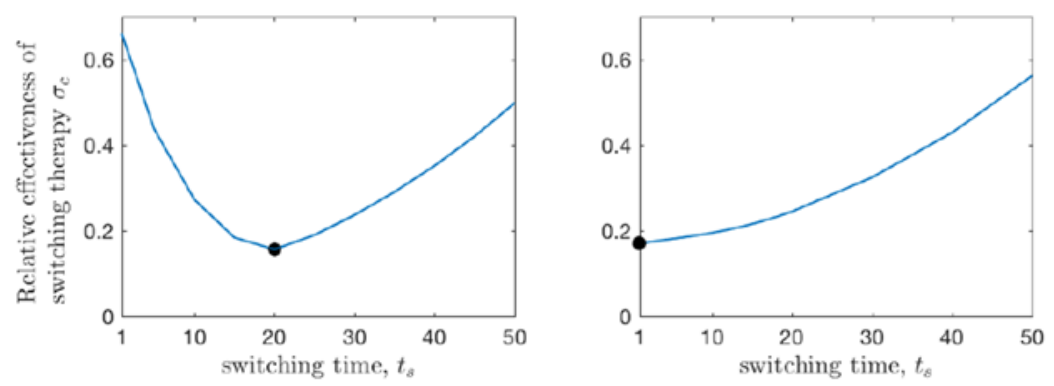
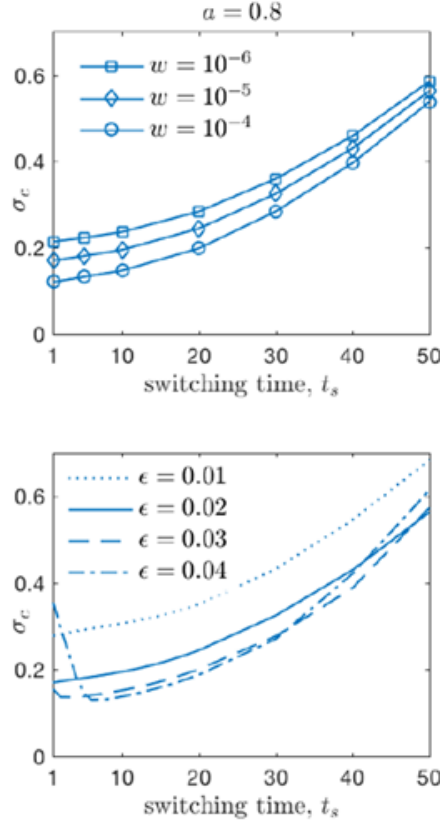

FiguRE 9. Number of cancer cells when the treatment begins with the cytotoxic drug at $t_{c}=10$, and then switches to the targeted drug at different times $(x)$. The relative effectiveness of switching therapy $\sigma_{c}$ is shown with $w=10^{-5}$ and $\epsilon=0.02$ (left), and compared for different values of $w$ and $\epsilon($ right). The targeted drug delays the relapse due to resistance to the cytotoxic drug. In particular, when $a=0.2$, there exists a switching time that minimizes the number of cancer cells, approximately $t_{s}=20$. However, in the more competitive case, $a=0.8$, it is better to switch the drugs earlier since the targeted drug is more effective, unless there are many preexisting cells that are resistant to the targeted drug $(\epsilon \geq 0.04)$.

and (ii) a linear model for which the outcome is similar to a discrete two-states model,

$$
r_{c}(\theta)=\left(\eta_{c}-\frac{\eta_{c}}{4}\right) \prod_{i=1}^{2}\left(1-\theta_{i}\right)+\frac{\eta_{c}}{4}, \quad \mu_{c}(\theta)=0.8\left(1-\theta_{1}\right), \quad \varphi_{c}(\theta)=0.8\left(1-\theta_{2}\right),
$$

and similarly in the model for the healthy cells. We remark that a larger class of functions can be classified into either one of the aforementioned cases based on concavity. For instance, when either proliferation function is concave up or the drug effect is concave down, the outcome shows discrete state, similar to the linear model. For more detail, see [15]. The parameters are taken to be comparable with equations (3.1)-(3.2). The death terms are assumed to be constant, $d_{h}(\theta)=d_{c}(\theta)=0.5$, and the epimutation rates are set as $\nu_{h}=\nu_{c}=10^{-3}$.

Figure 11 shows the distribution of both healthy cells, $n_{h}(t, \theta)$, and cancer cells, $n_{c}(t, \theta)$, in the resistance space. These results are computed with the quadratic and linear models, while increasing the drug dosage, $c_{i}=1,2,3,4$. The distributions are shown for the case when the competition allows cells to coexist $(a=0.2$ at time $t=80$ ). Figure 11 confirms that the quadratic model allows for intermediate resistance levels that gradually increase with increased drug dosage. As the dosages of the cytotoxic and targeted drugs increase, the mean resistance level of cancer cells $n_{c}(t, \theta)$ increases in the corresponding direction of $\theta_{1}$ and $\theta_{2}$. The resistance levels of healthy cells is affected only by the cytotoxic drug. In contrast to the smooth transition in the resistance level observed in the quadratic model, the outcome of the linear model is closer to binary 

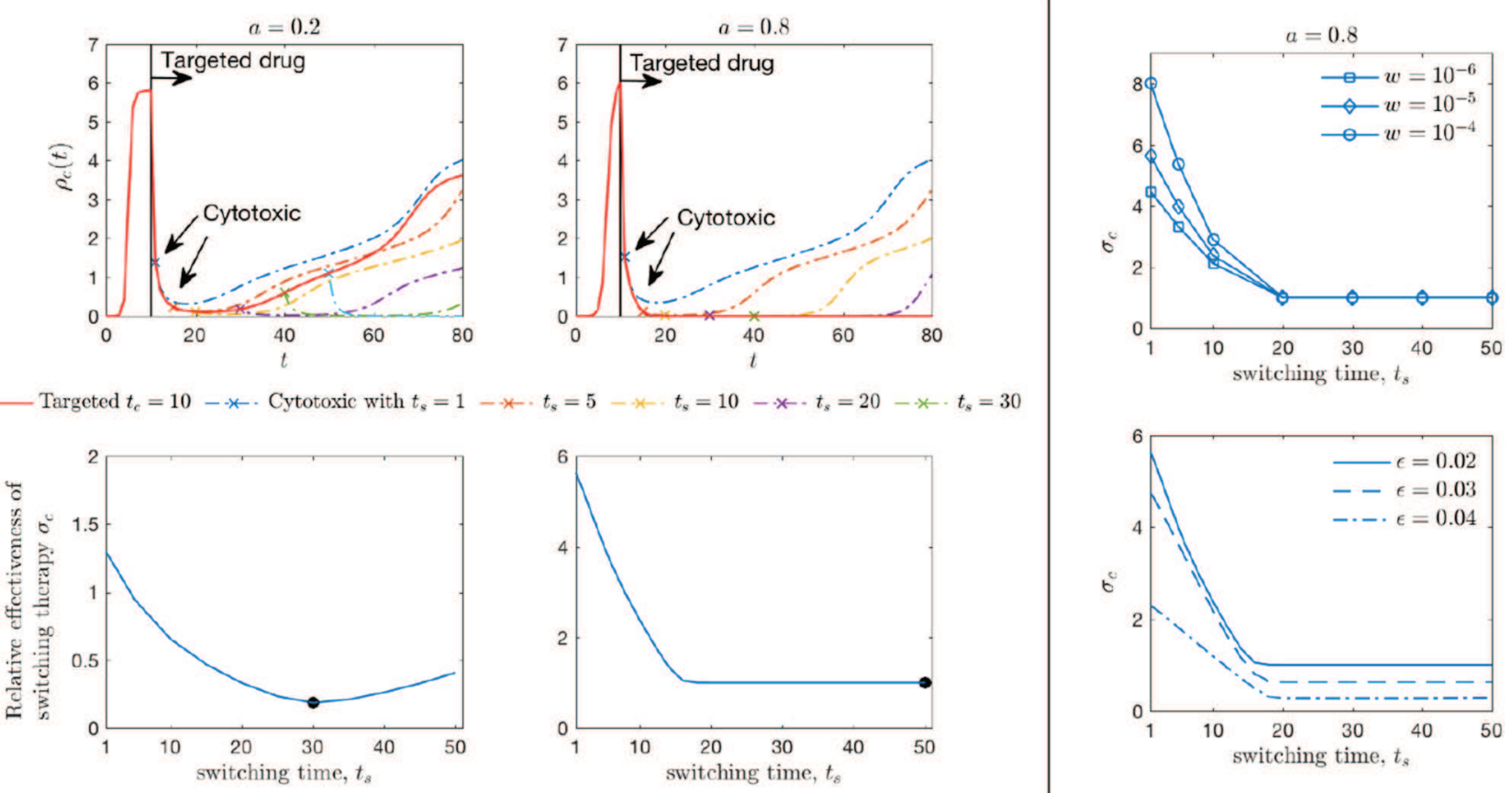

FiguRE 10. Number of cancer cells when the treatment begins with the targeted drug at $t_{c}=10$, and then switches to the cytotoxic drug at different times $(\times)$. The relative effectiveness of switching therapy $\sigma_{c}$ is shown with $w=10^{-5}$ and $\epsilon=0.02$ (left), and compared for different values of $w$ and $\epsilon$ (right). The cytotoxic drug delays the relapse due to targeted drug resistance when $a=0.2$. Switching the drug at $t_{s}=30$ minimizes the total number of cancer cells. When $a=0.8$, it is more effective to use the targeted drug only without switching to a cytotoxic drug.

as shown in Figure 11. With the dosage threshold of $c_{i} \approx 3$, the dominating resistance trait instantly changes from fully-sensitive $\left(\theta_{i}=0\right)$ to fully-resistant $\left(\theta_{i}=1\right)$. We note that in the highly competitive case, $a=0.8$, the distributions are similar to the results shown in Figure 11, only that the concentration of healthy cells is relatively low, $n_{h}(t, \theta) \approx 0$. The distinctive feature of the models can be analyzed by computing the maximal fitness trait $\theta_{c}^{*}=\operatorname{argmax}_{\theta}\left(r_{c}(\theta)-\mu_{c}(\theta) c_{1}-\varphi_{c}(\theta) c_{2}\right)$ depending on the drug dosages. In the quadratic model, the maximal fitness for the cytotoxic drug becomes $\theta_{c}^{*}\left(c_{1}, c_{2}=0\right)=\left(\mu c_{1} /\left(0.75 \eta_{c}+\mu c_{1}\right), 0\right)$ that gradually increases from 0 to 1 as $c_{1}$ increases. In the linear model, it is either $\theta_{c}^{*}=(0,0)$ or $\theta_{c}^{*}=(1,0)$ with threshold dosage $c_{1}=0.75 \eta_{c} / \mu$. The threshold in the linear model for our choice of parameters is $0.75 \eta_{c} / \mu \approx 2.8$, which agrees with the transition between $c_{i}=2$ and $c_{i}=3$ shown in Figure 11.

We proceed to studying the evolution of the distribution in the resistance space under drug switching therapy. The results are shown in the left columns of Figure 12 . Here, the initial drug with dosages satisfying $c_{1}+c_{2}=5$ is applied at $t_{c}=10$ and is then switched to the second drug at $t=20$. Prior to the treatment, the most sensitive cells dominate the population. However, after treatment is initiated, the resistant cells emerge, depending on the drug type. For instance, when the therapy is switched from a cytotoxic drug to a targeted drug, the distribution shifts from $\theta_{1} \gg 0$ and $\theta_{2}=0$ to $\theta_{2} \gg 0$. Compared with the outcome of a single drug therapy, the population of cancer cells that are resistant to one drug and sensitive to the other drug declines. Cells that are resistant to both drugs, $\theta_{1} \approx 1$ and $\theta_{2} \approx 1$, are likely to survive. We also study the reversal of drug sensitivity [67, 75] with the withdrawal of drugs and especially the effect of epimutation. The results are shown in the right column of Figure 12, where both drugs are applied with dosages $c_{1}=c_{2}=4$ at $t_{c}=10$ until $t=50$. The results show that the proliferation rate will be restored by the sensitive cells dominating the population after a certain time after 

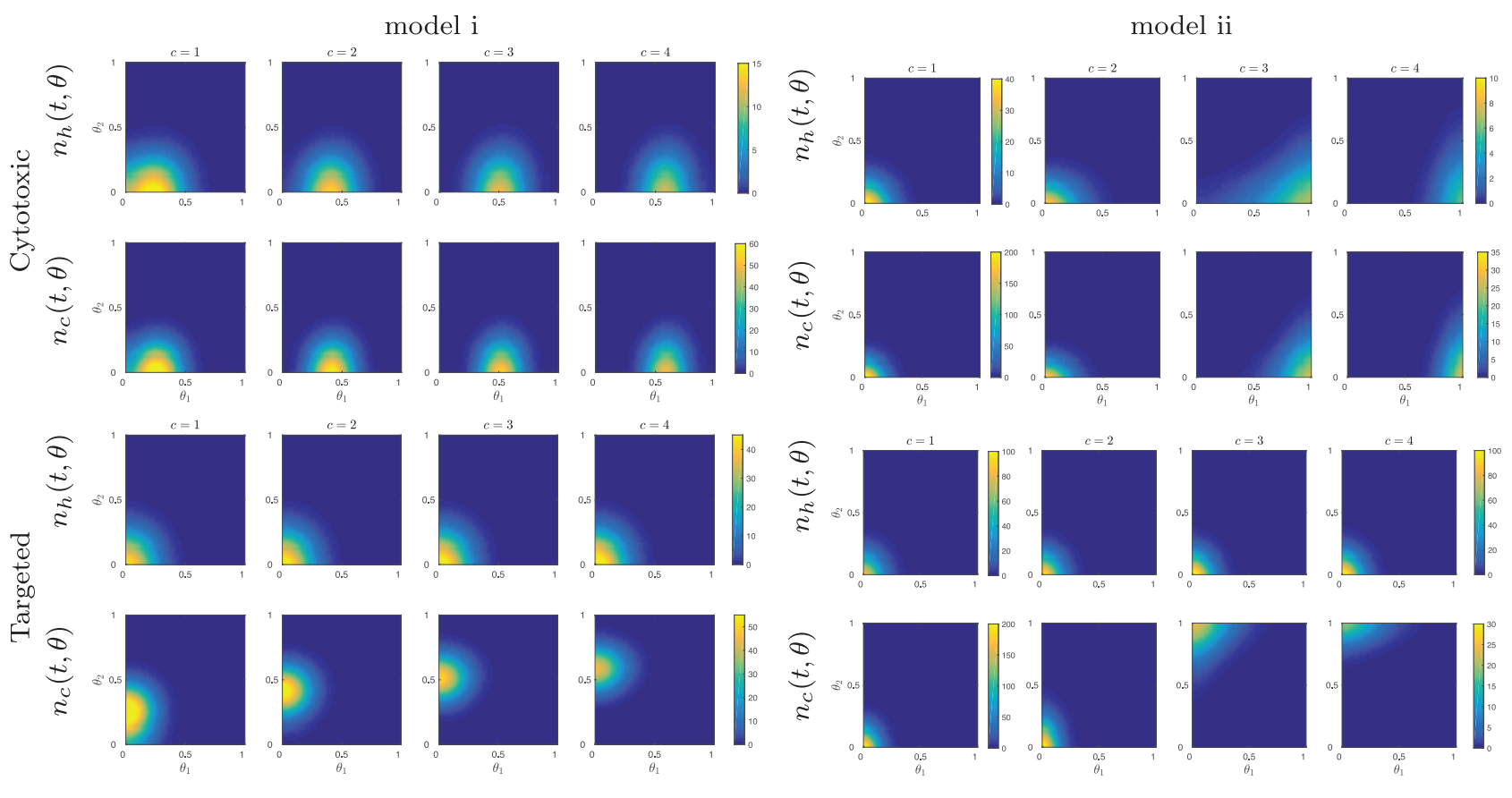

Figure 11. Distribution of healthy cells, $n_{h}(t, \theta)$, and cancer cells, $n_{c}(t, \theta)$, in the resistance phenotype space using a quadratic model (model i) and a linear model (model ii) with $a=0.2$ for different drug dosages $c_{i}=c=1,2,3,4$ at $t=80$. In the quadratic model, as the drug dosage increases, the mean resistance level in cancer cells gradually increases in the direction of $\theta_{1}$ or $\theta_{2}$ depending on the drug type. On the other hand, in linear model the level of resistance in cancer cells change from fully-sensitive, $\theta_{i}=0$, to fully-resistant, $\theta_{i}=1$, depending on the drug dosage. This is a sharp transition compared with the quadratic model. Healthy cells are only affected by the cytotoxic drug.

the drugs are withdrawn. Moreover, epimutation accelerates this process. Drug sensitivity is reversed around $t=80$ when epimutation rate is $\nu_{c}=10^{-3}$, but that is not the case when $\nu_{c}=10^{-4}$, where the distributions at $t=100$ are not yet reversed. The restoration completes around $t=130$ for the case $\nu_{c}=10^{-4}$. This is due to the effect of epimutation increasing heterogeneity, that can be observed in larger variances of cell distributions when $\nu_{c}=10^{-3}$ compared to $\nu_{c}=10^{-4}$.

Figure 13 compares the effect of switching therapy to a single drug therapy for the two continuum models. The relative size of the total cancer cell population compared with a single (cytotoxic) drug therapy, is $\int_{10}^{100} \rho_{c}(t) \mathrm{d} t / \int_{10}^{100} \rho_{c}^{*}(t) \mathrm{d} t$, where $\rho_{c}^{*}(t)$ is the number of cancer cells under a cytotoxic drug treatment. Once again, the results confirm that a single targeted drug therapy is particularly effective when the cells are highly competitive, $a=0.8$. This is more significant in the linear model, where the relative size of the cancer cell population reduces approximately to $50 \%$, compared with $80 \%$ with the quadratic model. When $a=0.2$, the switching therapy in any order is more effective than the single drug therapies regardless of the continuum model $\left(t_{s} \geq 5\right)$. We note that the outcome of the linear model strongly depends on the switching time $t_{s}$, and particularly becomes worse than the single targeted drug therapy when $a=0.8$, if the tumor is not exposed to the targeted drug for a sufficiently long period.

\subsection{Alternating therapies and on-off combination therapies}

In the previous sections we studied the emerging dynamics when switching cytotoxic and targeted drugs. The study was performed assuming competition between healthy cells and cancer cells, and a continuum resistance 

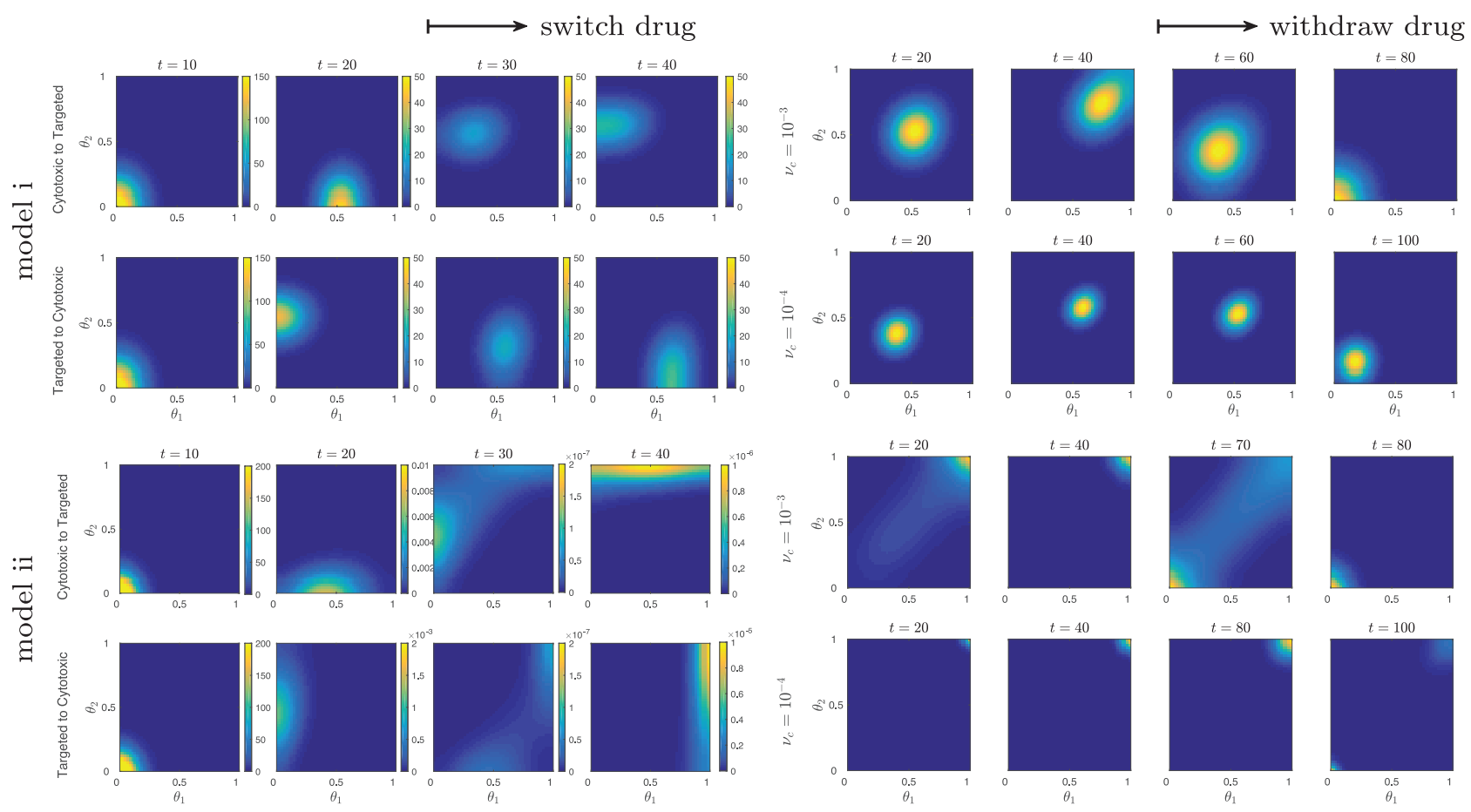

$t=20$
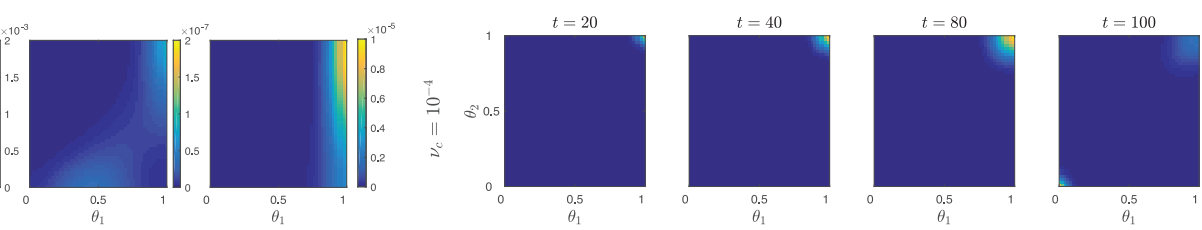

Figure 12. Evolution of the cancer cell distribution $n_{c}(t, \theta)$ at $t=10,20,30,40$ using the quadratic model (top) and the linear model (bottom) with a drug switching therapy (left) and withdrawal of drugs (right). The initial drug is applied at $t_{c}=10$, and the second drug is applied at $t=20$ using the switching therapy. In contrast to the single drug therapy, a combination therapy reduces the levels of cancer cells that are resistant to one drug yet are sensitive to the other drug. The right figures show the restoration of drug sensitivity by the withdrawal of drugs at $t=50$, where both drugs are applied at $t_{c}=10$. The epimutation accelerates the restoration process, where the sensitive population is restored at $t=80$ when the epimutation rate $\nu_{c}=10^{-3}$, but not for smaller epimutation $\nu_{c}=10^{-4}$.

trait. In this section, we assume that the drug can be changed within a short period of time, and study the periodically alternating therapy and the on-off combination schedules depicted in Figure 4c,d. The results for these studies are shown in Figures 14. In both figures, the drug therapies start at $t_{c}=10$. We test for different dosages: (i) a moderate dosage $c_{1}+c_{2}=3$ aiming at maintaining the cancer cell population at low levels; and (ii) a high dosage, $c_{1}+c_{2}=5$, aiming at completely eliminating the cancer cells. We also test for different competition rates $a=0.2$ and 0.8 . As a reference, we plot the results obtained with a single drug therapy.

Figure 14 shows the dynamics of the cancer cells under the moderate dosage. We observe that the alternating therapy is more effective than the other therapies, as it reduces the overall number of cancer cells compared to single drug therapies. In particular, alternating the drug with a short period $\left(t_{p}=2\right)$ suppresses the cancer growth without oscillations. Initiating the alternating therapy in any order ends with similar results for both competition rates since the preexisting resistant populations to both drugs are identical. On the other hand, the on-off combination therapy yields a highly oscillatory behavior that results in a larger numbers of cancer cells during the off period compared with the single drug therapy. Since the dosage of chemotherapy is restricted due to its toxic nature $[18,73]$, the on-off combination therapy may not be effective in such situations.

In the case of a higher dosage, $c_{1}+c_{2}=5$, we observe that the number of cancer cells reduces to less than an order of magnitude for a certain time period before a relapse occurs. As shown in Figure 14, both of the 

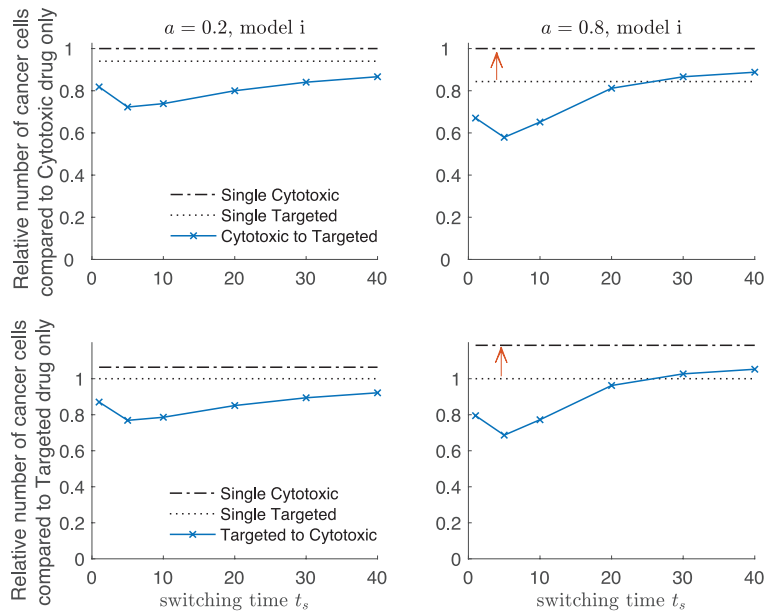
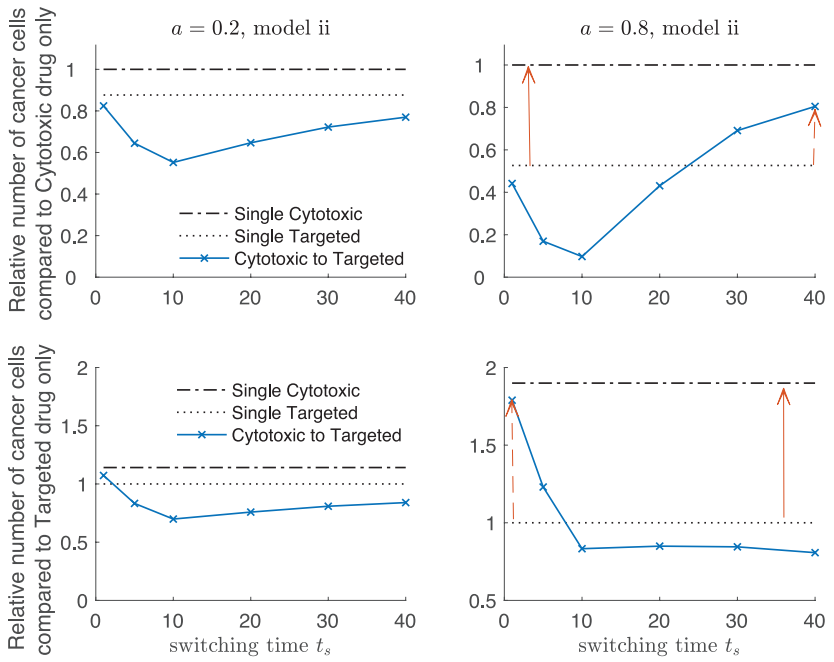

FIGURE 13. Relative number of cancer cells using drug switching therapy compared to using a single cytotoxic drug for different values of drug switching time $t_{s}$. The results are shown for the quadratic model (top) and the linear model (bottom). The linear model is more sensitive to the switching time, and when $a=0.8$, the outcome of a single cytotoxic drug therapy or the switching therapy, can be worse than a single targeted drug therapy, where the arrows indicate the increased amount.

alternating and combination schedules significantly delay the relapse compared to the single drug therapies. As before, alternating the drug with shorter periods $\left(t_{p}=2\right)$ keeps the cancer population below a certain threshold, in contrast to longer periods that may result in small peaks throughout the treatment. However, when $a=0.8$, we observe that initiating the therapy with the targeted drug is more effective in the sense that it overcomes the drawback of longer drug periods. We also observe that the on-off combination therapy with a high dosage effectively reduces the cancer cells population and delays the relapse, similarly to the alternating schedule with short periods. However, after the relapse, the cancer cell population fluctuates more than it does with the alternating schedule.

The results obtained so far assume equal sizes of preexisting populations that are resistant to the cytotoxic and to the targeted drugs. However, since the preexisting resistance is one of the critical factor in relapse, we further study the more realistic scenario in which different fractions of the pre-treatment population are resistant to both drugs. We denote the number of cells that are resistant to the cytotoxic drug and to the targeted drug as

$$
\rho_{c, R 1}(t)=\int_{\mathbf{1}_{\theta_{1} \geq 0.5}} n_{c}(t, \theta) \mathrm{d} \theta, \quad \rho_{c, R 2}(t)=\int_{\mathbf{1}_{\theta_{2} \geq 0.5}} n_{c}(t, \theta) \mathrm{d} \theta
$$

respectively. We remark that the previous results correspond to the case $\rho_{c, R 1}(0)=\rho_{c, R 2}(0)$. We consider the initial condition as in equation (3.3), but with different variances $\epsilon=0.02$ or 0.08 for each direction, $\theta_{1}$ or $\theta_{2}$. The ratio then either becomes $\rho_{c, R 1}(0) / \rho_{c, R 2}(0)=10^{-4}$ or $\rho_{c, R 1}(0) / \rho_{c, R 2}(0)=10^{4}$.

Figure 15 presents the results for the case when the cytotoxic resistant cells have a higher ratio, that is, $\rho_{c, R 1}(0)>\rho_{c, R 2}(0)$. We test the same therapies as before including the single drug, alternating therapies, and combination therapies. We consider the moderate dosage $c_{1}+c_{2}=3$ and the high dosage $c_{1}+c_{2}=5$. The competition rates are set as $a=0.2$ and 0.8 . Similar conclusions hold as in the symmetric pre-treatment case. With a relatively low dosage, the alternating schedule with a small period works remarkably better than the combination therapy, while with a relatively high dosage, the combination therapy can also suppress the tumor 

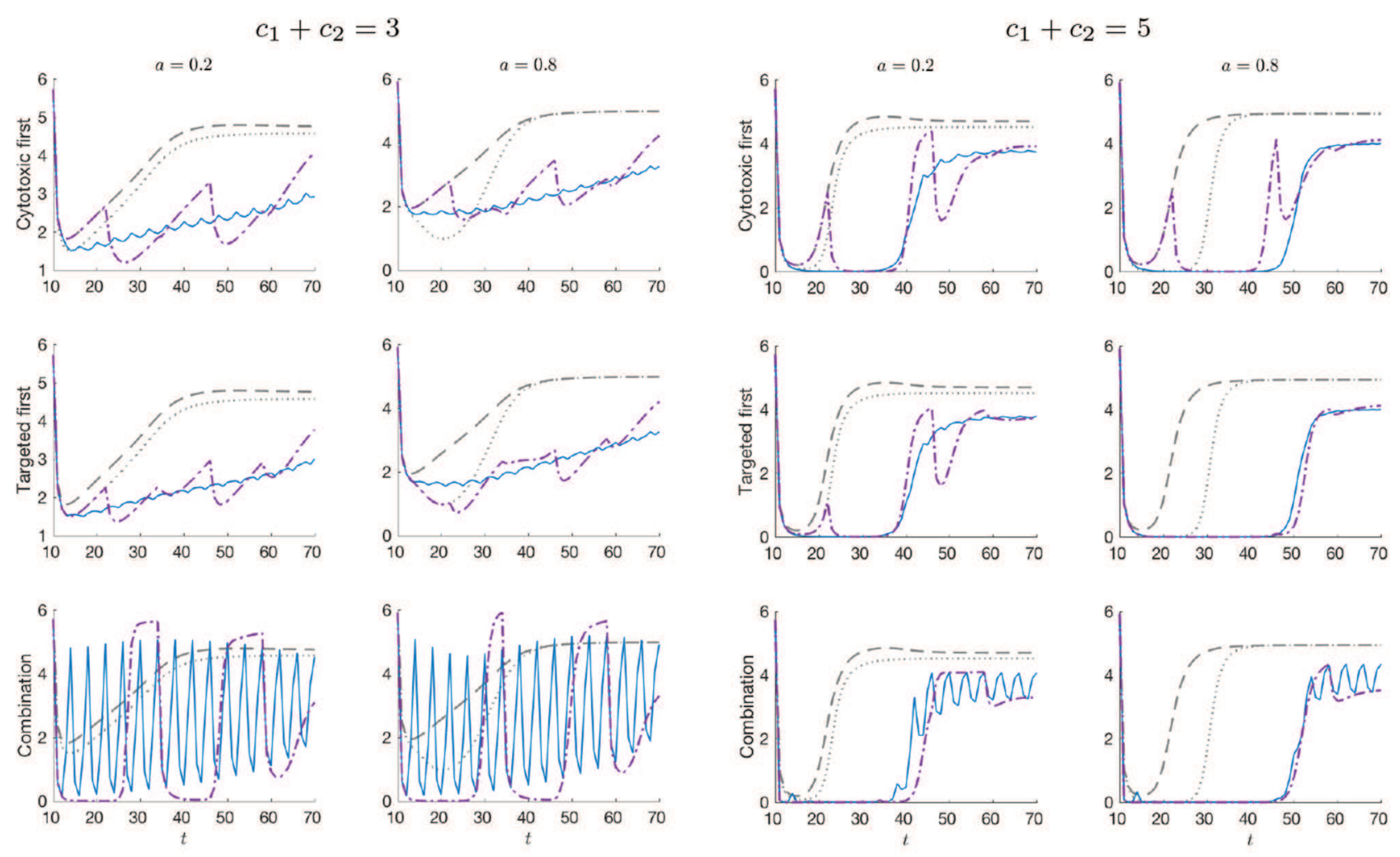

Single drug therapy

Alternating or combination therapy

$$
\begin{gathered}
--c_{1}>0, c_{2}=0 \cdots \cdots \cdot c_{1}=0, c_{2}>0 \\
-t_{p}=2 \quad-\cdot-\cdot t_{p}=12
\end{gathered}
$$

FIgURE 14. The number of cancer cells $\rho_{c}(t)$ using different therapies including single-drug, alternating, and combination therapy with a relatively low dosage $c_{1}+c_{2}=3$ and a relatively high dosage $c_{1}+c_{2}=5$. The periods of alternating and combination therapies are taken as $t_{p}=2,5,8$, and 12 . The alternating therapy in any order is more effective than the other approaches considering the overall number of cancer cells during the treatment. A shorter alternating period $\left(t_{p}=2\right)$ suppresses the cancer cells without oscillations. On the other hand, the on-off combination therapy yields a highly oscillatory outcome. In case of $a=0.8$ using high dosage, initiating the therapy with the targeted drug is more effective than initiating it with the cytotoxic drug, and the on-off combination therapy also delays the relapse effectively.

growth for a certain period of time. However, in the asymmetric pre-treatment case, the order of drugs becomes more important to the therapy outcome. First, the outcome of single drug therapy is correlated with the size of the preexisting resistance: In Figure 15, the cytotoxic drug produces a worse outcome due to a larger pretreatment resistant population, while we remark that the targeted drug yields the early relapse portrayed in the opposite case $\rho_{c, R 1}(0)<\rho_{c, R 2}(0)$. In addition, when $\rho_{c, R 1}(0)>\rho_{c, R 2}(0)$, initiating an alternating schedule with a targeted drug is more effective than initiating it with the cytotoxic drug, particularly for higher dosages. Clearly, this is the outcome because the targeted drug reduces the population of cells that are resistant to the cytotoxic drug. In addition, for the highly competitive case, $a=0.8$, we observe that a single targeted drug therapy with dosage $c_{1}+c_{2}=3$ yields the minimal number of cancer cells up to $t \approx 40$ with a relatively low dosage. This provides us with an opportunity to design an effective adaptive therapy.

On the other hand, when $\rho_{c, R 1}(0)<\rho_{c, R 2}(0)$, it is better to initiate the treatment with the cytotoxic drug. As expected, the results suggest that the pretreatment drug resistance can be a critical factor in determining 

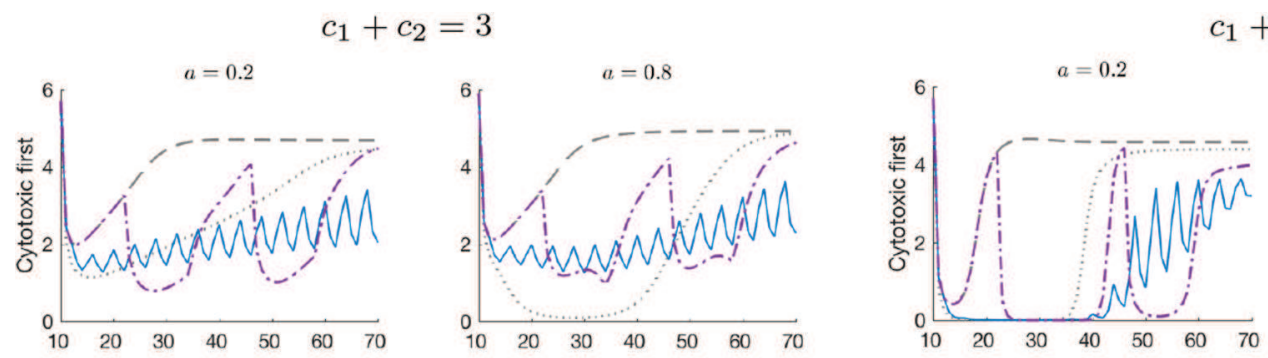

$$
c_{1}+c_{2}=5
$$
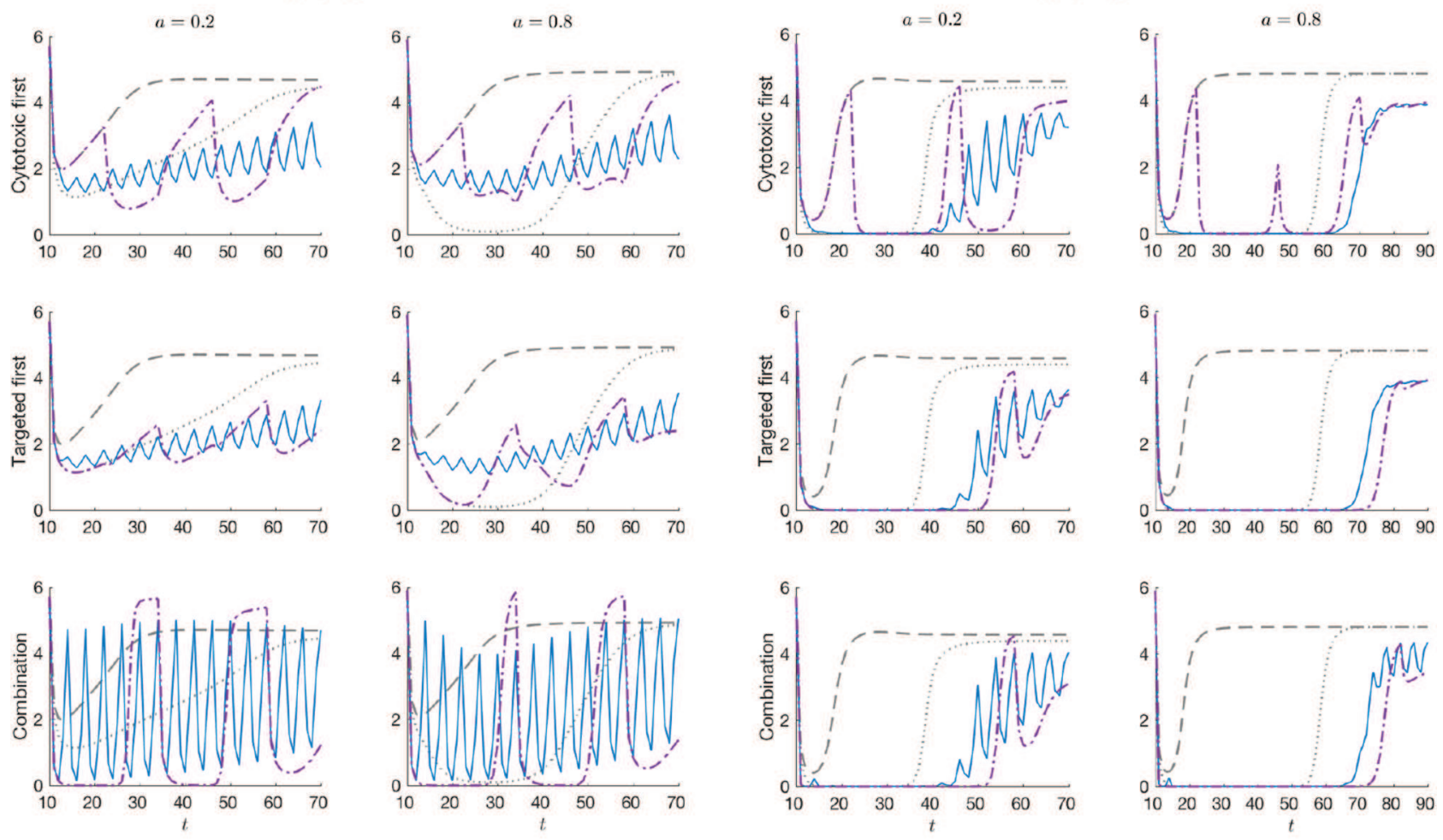

Single drug therapy

$$
\begin{gathered}
--c_{1}>0, c_{2}=0 \cdots \cdots \cdot c_{1}=0, c_{2}>0 \\
-t_{p}=2 \quad-\cdot-\cdot t_{p}=12
\end{gathered}
$$

Figure 15. Number of cancer cells $\rho_{c}(t)$ for different therapies when the number of cells that are resistant to the cytotoxic drug is larger than those that are resistant to the targeted drug $\left(\rho_{c, R 1}>\rho_{c, R 2}\right)$. We observe that initiating the alternating therapy with the targeted drug with a smaller resistant population is more effective.

the course of therapy and its outcome. Moreover, this result differs from the one obtained in [66], in which a combination therapy of cytotoxic chemotherapy and immunotherapy assuming resistance to only cytotoxic drugs was considered. When cells that are resistant to cytotoxic drugs are present, it was suggested to first apply the cytotoxic drug [66]. Although in clinical settings, it is difficult evaluate the pretreatment resistance levels for different drugs, the results suggest that it is important develop methodologies to adjust the therapy to the pretreatment resistance levels.

In addition to drug scheduling, we also study the effect of dosages with respect to asymmetric preexisting resistance. In Figure 16, we present the number of cancer cells $\rho_{c}(t)$ at $t=100$ using a combination therapy with a cytotoxic drug dosage $c_{1}$ and a targeted drug dosage $c_{2}$. When $\rho_{c, R 1}>\rho_{c, R 2}$, we observe that a higher dosage of the targeted drug is more effective than increasing the dosage of the cytotoxic drug. For instance, the dosage $\left(c_{1}, c_{2}\right)=(2,3)$ results in a smaller tumor than $\left(c_{1}, c_{2}\right)=(3,2)$. In the opposite case, $\rho_{c, R 1}<\rho_{c, R 2}$, the result is reversed: $c_{1}<c_{2}$ is a more effective treatment. When the competition is mild, $a=0.2$, increasing the dosages of both drugs constantly improves the outcome. However in the highly competitive case, $a=0.8$, there exists an optimal dosage of the cytotoxic drug. For instance, when $\rho_{c, R 1}>\rho_{c, R 2},\left(c_{1}, c_{2}\right)=(1.5,3)$ results with the minimum number of cancer cells. The optimal dosage changes to $\left(c_{1}, c_{2}\right)=(2.5,3)$, with a slightly larger cytotoxic drug dosage when $\rho_{c, R 1}<\rho_{c, R 2}$. 

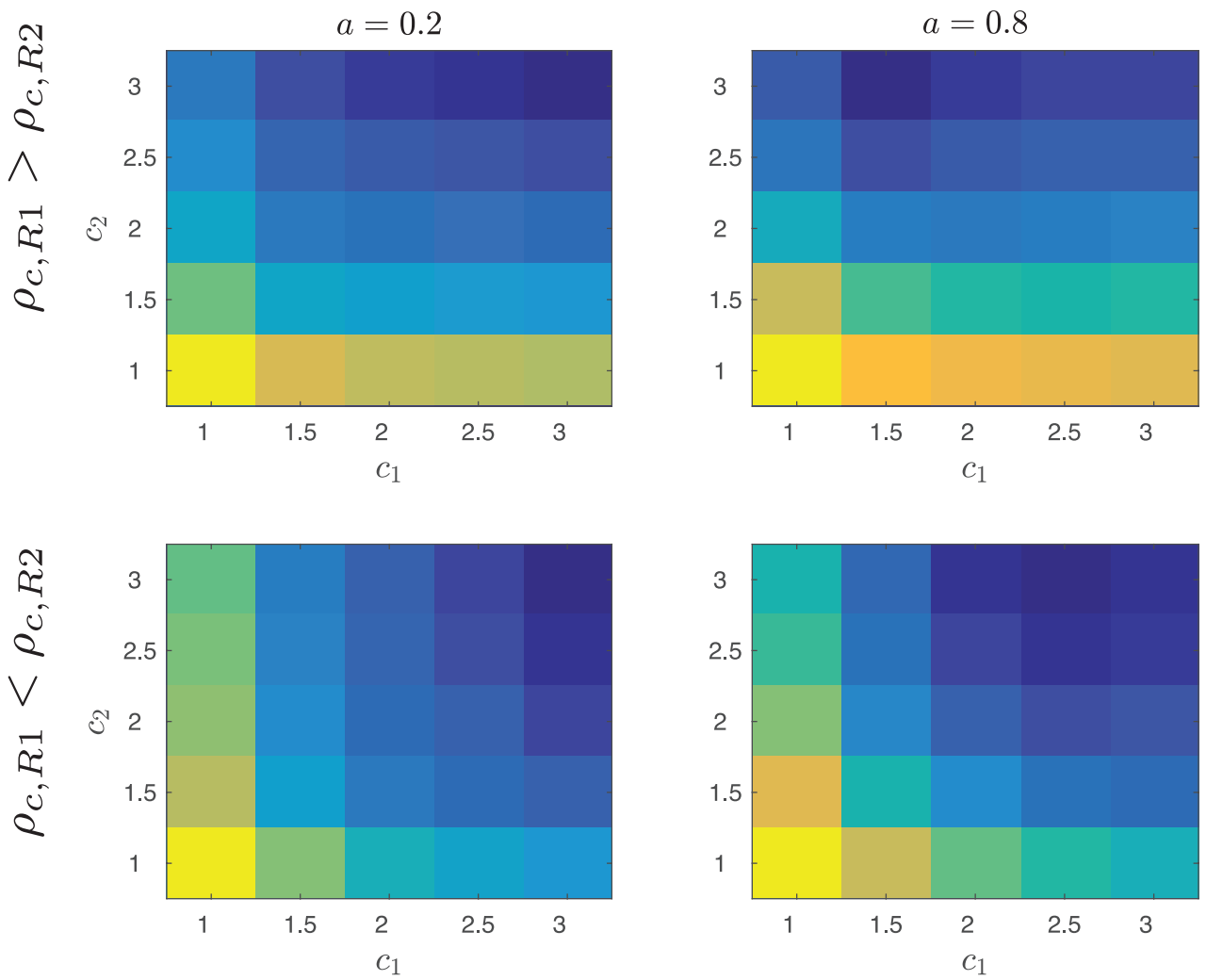

Figure 16. Number of cancer cells at time $t=100, \rho_{c}(100)$, for different dosages of combination therapy involving cytotoxic and targeted drugs. The results are tested for different sizes of preexisting resistance either $\rho_{c, R 1}<\rho_{c, R 2}$ or $\rho_{c, R 1}>\rho_{c, R 2}$. As expected, initiating the switching therapy with the drug that has a smaller resistant population is more effective. When $a=0.8$, there exists an optimal dosage of the cytotoxic drug.

\section{TUMOR GROWTH MODEL WITH CELL COMPETITION}

We extend the competition model by including a space variable $x \in[-1,1]^{2} \subset \mathbb{R}^{2}$. The concentrations of healthy cells, $n_{h}(t, x, \theta)$, and cancer cells, $n_{c}(t, x, \theta)$, are governed by the following system,

$$
\begin{aligned}
& \partial_{t} n_{h}(t, x, \theta)=G_{h} n_{h}+\nu_{h} \Delta_{\theta} n_{h}+\nu_{n} \Delta_{x} n_{h}+\nu_{p} \nabla_{x} \cdot\left(n_{h} \nabla_{x} P_{h}\right), \\
& \partial_{t} n_{c}(t, x, \theta)=G_{c} n_{c}+\nu_{c} \Delta_{\theta} n_{c}+\nu_{n} \Delta_{x} n_{c}+\nu_{p} \nabla_{x} \cdot\left(n_{c} \nabla_{x} P_{c}\right) .
\end{aligned}
$$

Here, $P_{h}(t, x)=\left(\rho_{h} / \rho_{h, 0}\right)^{k}$ and $P_{c}(t, x)=\left(\rho_{c} / \rho_{c, 0}\right)^{k}$ are the cell pressures for the healthy cells and the cancer cells, respectively, where the total number of cells $\rho_{h}(t, x)$ and $\rho_{c}(t, x)$ is given by equation (2.3). The normalization constants are taken as the maximum cell capacity $\rho_{h, 0}=3$ and $\rho_{c, 0}=6$. The growth terms, $G_{h}$ and $G_{c}$, and the epimutation terms with the operator $\Delta_{\theta}$, are taken as in equations (2.1) and (2.2), with the parameters chosen as in Section 3.1 and $\nu_{h}=\nu_{c}=10^{-4}$. The spatial competition model follows the tumor growth model developed in [14], where $\nu_{n}$ and $\nu_{p}$ are constants describing cell motility, that adopts the Hele-Shaw type tumor growth models [70]. The cell motility parameters are taken as $\nu_{n}=10^{-6}, \nu_{p}=10^{-5}$, and $k=6$ [7]. The boundary conditions in the physical space are taken as Neumann conditions with zero normal derivative just like in the phenotypic space. 

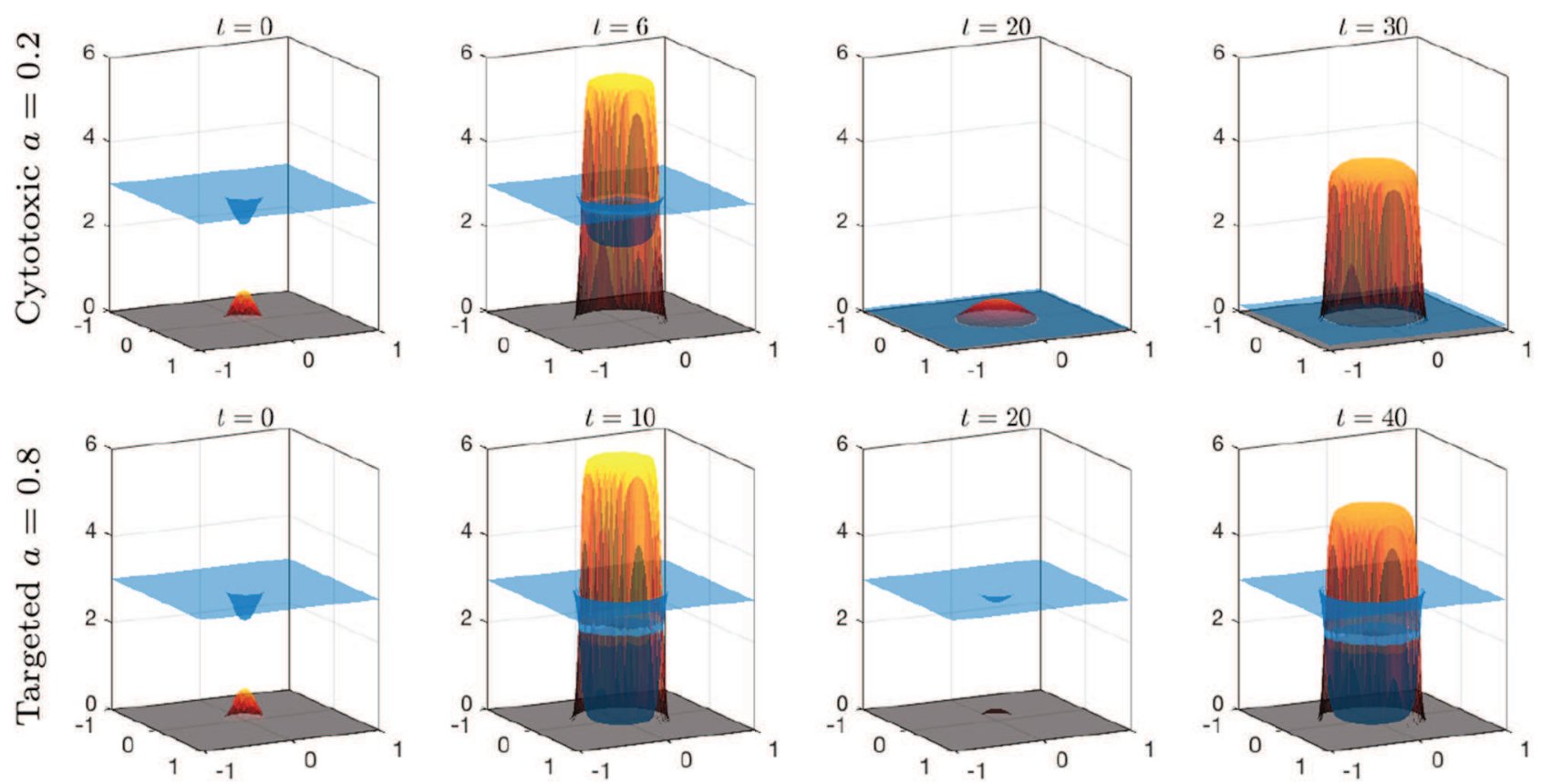

FiguRE 17. The evolution of cancer cells $\rho_{c}(t, x, y)$ and healthy cells $\rho_{h}(t, x, y)$ under singledrug therapies initiated with a small cancer population in the center of the domain $(l e f t, t=0)$. The cancer cells grow on top of the healthy tissue when $a=0.2$ (top, $t=6$ ). When $a=0.8$ (bottom, $t=10$ ), the cancer cells aggressively eliminate the healthy cells while expanding. The treatment starts at $t=6($ top $)$ and $t=10$ (bottom). The cancer cells relapse quickly when using a single drug therapy due to preexisting resistance.

We consider three spatially heterogeneous drug distributions to examine the therapies $c_{1}(t, x)$ and $c_{2}(t, x)$ :

i. A constant dosage,

$$
c_{i}(t, x)=\bar{c}_{i}(t) .
$$

ii. A diffusive case, where the drug diffuses from the right edge $x_{1}=1$ [63],

$$
c_{i}(t, x)=\bar{c}_{i}(t)\left[\left(e^{\lambda\left(x_{1}+1\right) / 2}+e^{-\lambda\left(x_{1}+1\right) / 2}\right) /\left(e^{\lambda}+e^{-\lambda}\right)\right]
$$

with $\lambda=\sqrt{2}$.

iii. A highly heterogeneous case [71],

$$
\begin{aligned}
c_{i}(t, x)= & \bar{c}_{i}(t)\left[2+0.25 \sin \left(2 \pi\left\|\left(x_{1}+1, x_{2}+1\right)\right\|_{2}\right)\right. \\
& \left.+0.5 \sin \left(4 \pi\left\|\left(1-x_{1}, x_{2}+1\right)\right\|_{2}\right)\right] / 2.75 .
\end{aligned}
$$

We assume a similar dependence of the proliferation on the space variable with all three cases considered. We choose the initial condition as a small concentration of cancer cells embedded in the center of a healthy tissue (see Fig. 17, $t=0)$. Preexisting resistance is set by taking $\epsilon=0.05$. The following results are presented by plotting the total number of healthy cells, $\rho_{h}(t, x, y)=\int n_{h}(t, x, y, \theta) \mathrm{d} \theta$, and cancer cells, $\rho_{c}(t, x, y)=\int n_{c}(t, x, y, \theta) \mathrm{d} \theta$. 

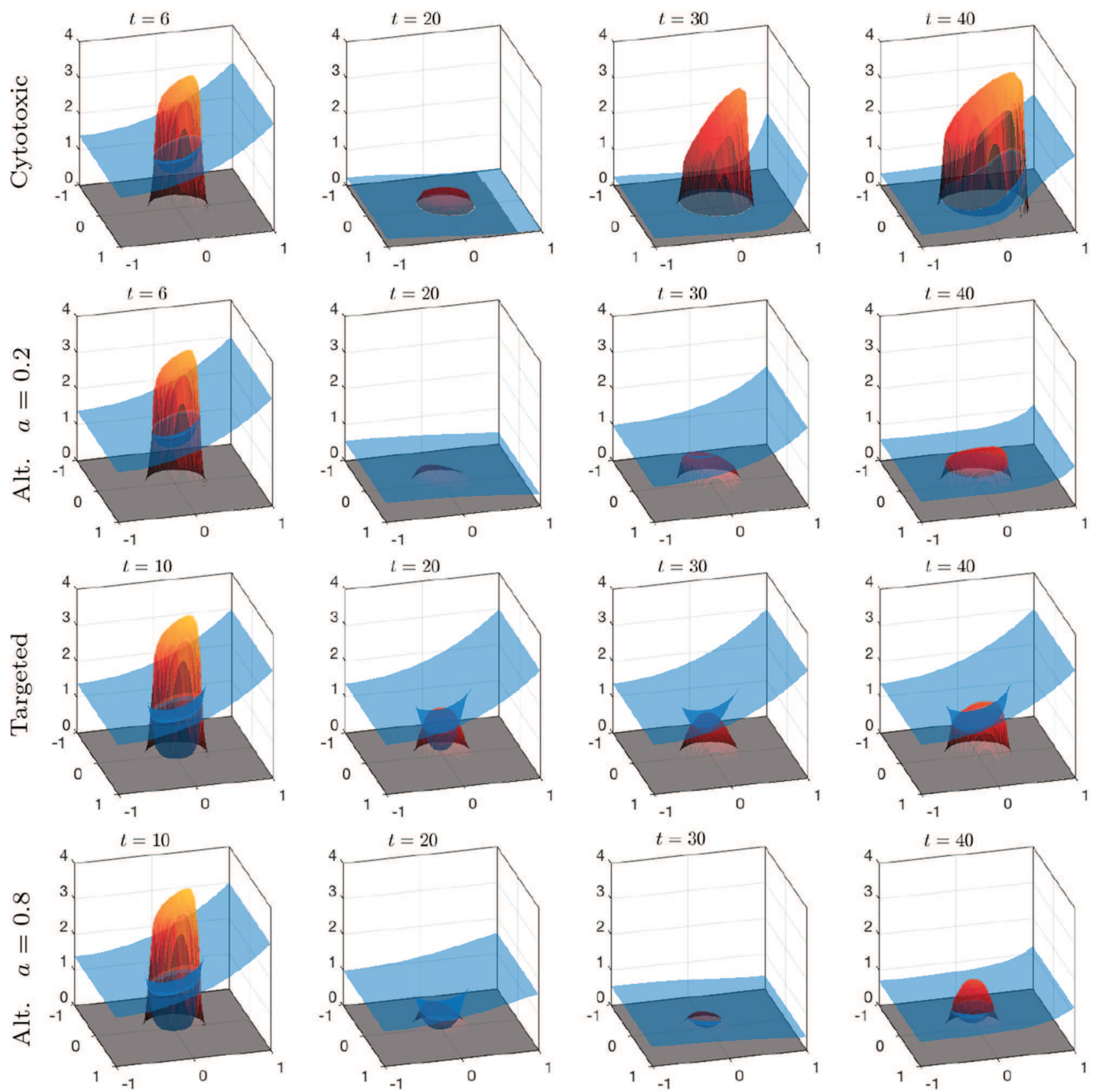

FiguRE 18. The evolution of cancer cells $\rho_{c}(t, x, y)$ and healthy cells $\rho_{h}(t, x, y)$ under singledrug and alternating (Alt.) therapies diffused from the right boundary, $x=1$. When $a=0.2$, the alternating therapy is remarkably effective compared with a single cytotoxic drug. When $a=0.8$, a single targeted drug therapy is also effective, although the tumor size is slightly larger compared with the tumor size with the alternating therapy. 

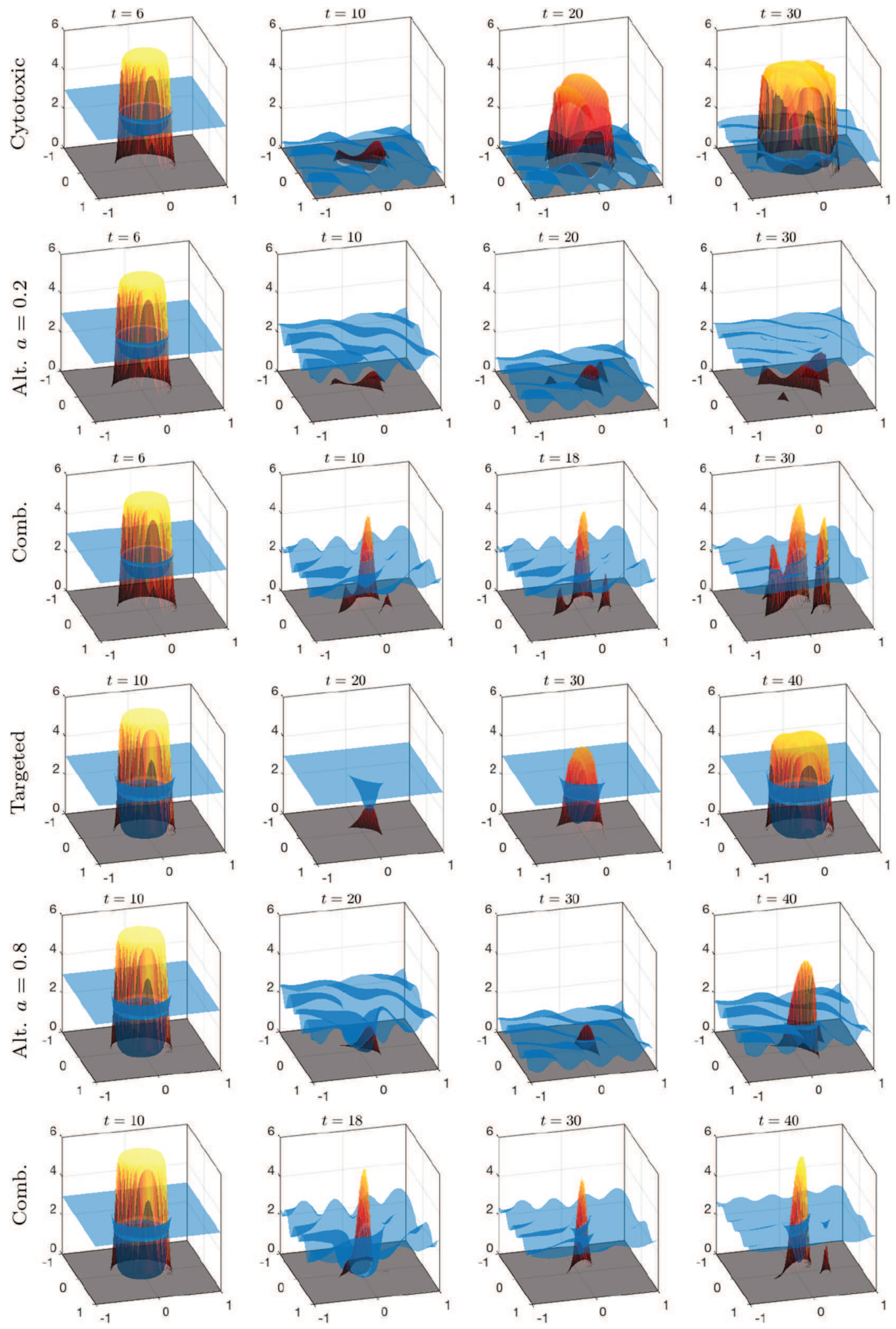

Figure 19. The evolution of cancer cells $\rho_{c}(t, x, y)$ and healthy cells $\rho_{h}(t, x, y)$ under different therapies with an irregular drug distribution. Local peaks of cancer cells can be observed that eventually grow into a tumor with a rough surface. The single-drug therapies result in strong relapses compared with the outcomes of alternating (Alt.) and combination (Comb.) therapies. Moreover, the alternating therapy is more effective than the on-off combination therapy particularly when $a=0.2$. 
Figure 17 corresponds to the case of a constant dosage. The initial tumor is located at the center of the domain. The first row shows the mildly competitive case, $a=0.2$, and treatment with the cytotoxic drug. The second row shows the highly competitive case, $a=0.8$, and treatment with the targeted drug. The treatments are initiated at $t_{c}=6$ and $t_{c}=10$, respectively, with dosages $\bar{c}_{1}=\bar{c}_{2}=5$. We observe that the cytotoxic therapy eliminates the healthy tissue in addition to the cancer cells, unlike the targeted drug. In addition, when $a=0.2$, the cancer cells grow on top of the healthy tissue, while in the highly competitive case, $a=0.8$, the cancer cells replace the healthy tissue while expanding. In both cases, a tumor treated with a single drug therapies quickly relapses due to the preexisting resistant cells. The spatial simulations with constant dosages are consistent with the results of the non-spatial model in the previous sections.

We now test combination therapies when the drug distribution is spatially heterogeneous. Figure 18 compares single drug therapies and alternating therapies with period $t_{p}=2$, when the resource and drugs are diffused from the right boundary, $x_{1}=1$. The treatment dosages are taken as $\bar{c}_{1}=7$ and $\bar{c}_{2}=4$. We remark that prior to the treatment, the tumor grows faster closer to the right boundary where the concentration of resources is high. Using a single drug therapy, the tumor relapses before $t=40$, particularly when $a=0.2$ using the cytotoxic drug. When $a=0.8$, we verify the effectiveness of the targeted drug, for which we observe that the size of the tumor at $t=40$ is smaller compared with the tumor at the same time using the cytotoxic drug, despite the lower drug dosage. The alternating therapies are more effective compared with the single-drug therapies in all competition environments, although the difference is smaller in the highly competitive case $a=0.8$, since the single targeted drug therapy is effective as well.

Finally, different therapies including the on-off combination therapies are compared in Figure 19. We set the dosages as $\bar{c}_{1}=\bar{c}_{2}=5$, which are sufficiently high so that the on-off combination therapies are effective as much as the alternating therapies. The drug distribution is heterogeneous. As expected, the single drug therapies result with strong relapses compared with the alternating and combination therapies. In addition, we observe emerging local peaks of cancer cells when using combination therapies during the off periods. This is particularly worse than the outcome of alternating therapies when $a=0.2$. In case of $a=0.8$, although the alternating therapy is more effective in suppressing the tumor throughout the treatment than the combination therapy, the sizes of the relapsed tumors at $t=40$ are similar.

\section{CONCLUSiON}

In this work we develop a competition model of healthy and cancer cells that takes into account resistance to cytotoxic and targeted drugs. We study the dynamics of resistance to the drugs and observe the emergence of populations with distinct levels of resistance depending on the therapy. Primarily, we classify the cell competition scenarios as either mild, where distinct cell types can coexist, or aggressive, where cancer cells dominate by actively eliminating the healthy cells. The threshold of the competition rate that distinguishes the two scenarios is related to the over-proliferation of the cancer cells over the healthy tissue. It also depends on the drug dosages. In addition, the analysis shows that targeted therapies have a greater potential of being effective when the cells are highly competitive.

Various drug treatments are tested in the two competition scenarios, and we observe that the treatment outcomes are distinctive. Although the targeted drug is more effective in the highly competitive case, using a single drug therapy, either cytotoxic or targeted, results with an eventual relapse due to the preexisting resistance, regardless of the strength of the competition. However, treatments that include both drugs show better outcomes in terms of the relapse time and the tumor size. Considering the drug switching therapy, an optimal switching time that minimizes the overall number of cancer cells exists when the competition is mild. In the highly competitive case, the targeted drug therapy alone is often effective enough. We also compare different continuum models that either allow for intermediate resistance states or are close to a two-state model with cells that are either fully sensitive or fully resistant to the drugs. Although the overall advantage of the switching therapy over single drug therapy in different competition environment holds, the linear model is shown to be more sensitive to the switching time that often yields a worse outcome compared with a single targeted drug therapy. Thus, when the population is highly competitive and the tumor proliferation and the 
drug effect linearly depend on the resistance trait, the drug switching time should be more carefully determined. Alternating treatments with different periods are shown to be effective in suppressing the cancer population during the entire treatment period compared to the other therapies. This particularly holds with small periods. Finally, we investigate a spatially heterogeneous tumor growth model, and verify that the same conclusions hold. We summarize our conclusions as follows, emphasizing that the effectiveness of chemotherapy and targeted drugs depends on the competition between populations and the drug resistance:

- Targeted drugs can be effective especially when the population is highly competitive $\left(a_{c h}\right.$ is large), and/or when resistance to chemotherapy has already been developed.

- A schedule to switch between chemotherapy and targeted cytotoxic drug can be optimized in mild environments.

- A relapse can be delayed by first administering the drug for which there is less preexisting resistance. Our results emphasize the importance of adjusting the therapy to the pretreatment resistance levels, especially when drug resistance is characterized by linear models.

As future work, we propose to incorporate experimental results considering combination of chemotherapy and targeted therapies [18, 73], also including cytostatic targeted drugs, and develop optimal strategies using optimal control theory for stabilizing the cancer population and/or minimizing the tumor size during the treatment period [11, 44, 69], while developing methodologies to take into account toxicity of targeted therapies to non-targets. Adaptive therapy is another interesting topic that aims at controlling the tumor by maintaining sensitive cells in order to suppress the resistant cancer cells [3,25]. Finally, the computational cost of simulating three-dimensional tumor growth models with multi-dimensional resistance traits is prohibitively expensive due to the high dimensionality. This requires developing an efficient numerical method that balances computational cost and accuracy $[16,35]$.

Acknowledgements. The work of DL was supported in part by the National Science Foundation under Grant Number DMS-1713109 and by the Jayne Koskinas Ted Giovanis Foundation.

\section{REFERENCES}

[1] R.A. Anderson and M. Chaplain, Continuous and discrete mathematical models of tumor-induced angiogenesis. Bull. Math. Biol. 60 (1998) 857-899.

[2] E.-a. D. Amir, L.K. Davis, D.M. Tadmor, F.E. Simonds, H. Levlne, Jacob, C.S. Bendall, K.D. Shenfeld, S. Krishnaswamy, P.G. Nolan and D. Pe'er, viSNE enables visualization of high dimensional single-cell data and reveals phenotypic heterogeneity of leukemia. Nat. Biotechnol. 31 (2013) 545-552.

[3] K. Bacevic, R. Noble, A. Soffar, O. Wael Ammar, B. Boszonyik, S. Prieto, C. Vincent, E.M. Hochberg, L. Krasinska and D. Fisher, Spatial competition constrains resistance to targeted cancer therapy. Nat. Commun. 8 (2017) 1-15.

[4] I. Bozic, T. Antal, H. Ohtsuki, H. Carter, D. Kim, S. Chen, et al., Accumulation of driver and passenger mutations during tumor progression. PNAS 107 (2010) 18545-18550.

[5] G.B. Birkhead, M.E. Rakin, S. Gallivan, L. Dones and D.R. Rubens, A mathematical model of the development of drug resistance to cancer chemotherapy. Eur. J. Cancer Clin. Oncol. 23 (1987) 1421-1427.

[6] R. Biswas, S. Gao, M.C. Cultraro, K.T. Maity, A. Venugopalan, Z. Abdullaev, K.A. Shaytan, A.C. Carter, A. Thomas, A. Rajan, Y. Song, et al., Genomic profiling of multiple sequentially acquired tumor metastatic sites from an "exceptional responder" lung adenocarcinoma patient reveals extensive genomic heterogeneity and novel somatic variants driving treatment response. Cold Spring Harbor molecular case studies 2 (2016) 1-26.

[7] D. Bray, Cell Movements: From Molecules to Motility, Garland Science, 2 edition (2000).

[8] A. Brock, H. Chang and S. Huang, Non-genetic heterogeneity - a mutation-independent driving force for the somatic evolution of tumours. Nat. Rev. Genet. 10 (2009) 336-342.

[9] A.H. Burris, S.H. Rugo, J.S. Vukelja, L.C. Vogel, A.R. Borson, S. Limentani, E. Tan-Chiu, E.I. Krop, A.R. Michaelson, et al., Phase II study of the antibody drug conjugate trastuzumab-DM1 for the treatment of human epidermal growth factor receptor 2 (HER2)-positive breast cancer after prior HER2-directed therapy. J. Clin. Oncol. 29 (2011) 398-405.

[10] A.L. Byers, L. Diao, J. Wang, P. Saintigny, L. Girard, M. Peyton, L. Shen, Y. Fan, U. Giri, K.P. Tumula, B.M. Nilsson, J. Gudikote, et al., An epithelial-mesenchymal transition gene signature predicts resistance to EGFR and PI3K inhibitors and identifies Axl as a therapeutic target for overcoming EGFR inhibitor resistance. Clin. Cancer Res. 19 (2013) $279-290$.

[11] C. Carrère, Optimization of an in vitro chemotherapy to avoid resistant tumours. J. Theor. Biol. 413 (2017) $24-33$.

[12] H.H. Chang, Y.P. Oh, E.D. Ingber and S. Huang, Multistable and multistep dynamics in neutrophil differentiation. BMC Cell Biol. 7 (2006) 1-12. 
[13] H. Cho and D. Levy, Modeling the dynamics of heterogeneity of solid tumors in response to chemotherapy. Bull. Math. Biol. 79 (2017) 2986-3012.

[14] H. Cho and D. Levy, Modeling the chemotherapy-induced selection of drug-resistant traits during tumor growth. J. Theor. Biol. 436 (2018) 120-134.

[15] H. Cho and D. Levy, Modeling continuous levels of resistance to multidrug therapy in cancer. Appl. Math. Model. 64 (2018) $733-751$.

[16] H. Cho, D. Venturi, E.G. Karniadakis, Numerical methods for high-dimensional probability density function equations. J. Comput. Phys. 305 (2016) 817-837.

[17] H.R. Chisholm, T. Lorenzi, A. Lorz, K.A. Larsen, N.D.L. Almeida, A. Escargueil and J. Clairambault, Emergence of Drug Tolerance in Cancer Cell Populations: An Evolutionary Outcome of Selection, Nongenetic Instability, and Stress-Induced Adaptation. Cancer Res. 75 (2015) 930-940.

[18] K. Dorris, C. Liu, D. Li, R.T. Hummel, X. Wang, J. Perentesis, O.M. Kim and M. Fouladi, A comparison of safety and efficacy of cytotoxic versus molecularly targeted drugs in pediatric phase I solid tumor oncology trials. Pediatr. Blood Cancer 64 (2017) $1-11$.

[19] T. Eichenlaub, S.M. Cohen and H. Herranz, Cell competition drives the formation of metastatic tumors in a Drosophila model of epithelial tumor formation. Curr. Biol. 26 (2016) 419-427.

[20] V. Fodal, M. Pierobon, L. Liotta and E. Petricoin, Mechanisms of cell adaptation: when and how do cancer cells develop chemoresistance? Cancer J. 17 (2011) 89-95.

[21] J. Foo and F. Michor, Evolution of acquired resistance to anti-cancer therapy. J. Theor. Biol. 355 (2014) 10-20.

[22] K. Fosgerau and T. Hoffmann, Peptide therapeutics: current status and future directions. Drug Discov. Today 20 (2015) $122-128$.

[23] K. Furugaki, I. Toshiki, S. Masatoshi, K. Kumiko, M. Yoichiro and M. Kazushige, Schedule-dependent antitumor activity of the combination with erlotinib and docetaxel in human non-small cell lung cancer cells with EGFR mutation, KRAS mutation or both wild-type EGFR and KRAS. Oncol. Rep. 24 (2010) 1141-1146.

[24] A.R. Gatenby and T.E. Gawlinski, A reaction-diffusion model of cancer invasion. Cancer Res. 56 (1996) $5745-5753$.

[25] A.R. Gatenby, S.A. Silva, J.R. Gillies and R.B. Frieden, Adaptive therapy. Cancer Res. 69 (2009) $4894-4903$.

[26] R. Glasspool, M.J. Teodoridis and R. Brown, Epigenetics as a mechanism driving polygenic clinical drug resistance. Br. J. Cancer 94 (2006) 1087-1092.

[27] B.P. Gupta, M.C. Fillmore, G. Jiang, D.S. Shapira, K. Tao, C. Kuperwasser and S.E. Lander, Stochastic state transitions give rise to phenotypic equilibrium in populations of cancer cells. Cell 146 (2011) 633-644.

[28] M.C. Garvey, E. Spiller, D. Lindsay, C.-T. Chiang, C.N. Choi, B.D. Agus, P. Mallick, J. Foo and M.S. Mumenthaler, A high-content image-based method for quantitatively studying context-dependent cell population dynamics. Sci Rep. 6 (2016) $1-12$.

[29] R. Gatenby and R. Gillies, A microenvironmental model of carcinogenesis. Nat. Rev. Cancer 8 (2008) 56-61.

[30] J. Gil and T. Rodriguez, Cancer: the transforming power of cell competition. Curr. Biol. 26 (2016) R164-R166.

[31] J.-P. Gillet and M.M. Gottesman, Mechanisms of multidrug resistance in cancer. Methods Mol. Biol. 596 (2010) $47-76$.

[32] B.S. Goldberg, R.G. Oxnard, S. Digumarthy, A. Muzikansky, M.D. Jackman, T.I. Lennes and V.L. Sequist, Chemotherapy with Erlotinib or chemotherapy alone in advanced non-small cell lung cancer with acquired resistance to EGFR tyrosine kinase inhibitors. Oncologist 18 (2013) 1214-1220.

[33] M.M. Gottesman, Mechanisms of cancer drug resistance. Annu. Rev. Med. 53 (2002) 615-627.

[34] M.M. Gottesman, T. Fojo and S.E. Bates, Multidrug resistance in cancer: role of ATP-dependent transporters. Nat. Rev. Cancer 2 (2002) 48-58.

[35] L. Grasedyck, D. Kressner and C. Tobler, A literature survey of low-rank tensor approximation techniques. GAMM Mitteilungen 36 (2013) 53-78.

[36] J. Greene, O. Lavi, M.M. Gottesman and D. Levy, The impact of cell density and mutations in a model of multidrug resistance in solid tumors. Bull. Math. Biol. 74 (2014) 627-653.

[37] D. Hanahan and R.A. Weinberg, Hallmarks of cancer: the next generation. Cell 144 (2011) 646-674.

[38] G. Housman, S. Byler, S. Heerboth, K. Lapinska, M. Longacre, N. Snyder and S. Sarkar, Drug resistance in cancer : An Overview. Cancers 6 (2014) 1769-1792.

[39] T. Hillen, M. Lewis, Managing Complexity, Reducing Perplexity - Modeling biological systems. Springer 13-25.

[40] Y. Iwasa, A.M. Nowak and F. Michor, Evolution of resistance during clonal expansion. Genetics 172 (2006) $2557-2566$.

[41] S. Jones, W. Chen, G. Parmigiani, F. Diehl, N. Beerenwinkel, T. Antal, et al., Comparative lesion sequencing provides insights into tumor evolution. PNAS 105 (2008) 4283-4288.

[42] Y. Jiang, Q. Yuan and Q. Fang, Schedule-dependent synergistic interaction between docetaxel and gefitinib in NSCLC cell lines regardless of the mutation status of EGFR and KRAS and its molecular mechanisms. J. Cancer Res. Clin. Oncol. 140 (2014) 1087-1095.

[43] R. Levayer, Cell competition: How to take over the space left by your neighbours. Curr. Biol. 28 (2018) R741-R744.

[44] D.V. Jonsson, M.C. Blakely, L. Lin, S. Asthana, N. Matni, V. Olivas, E. Pazarentzos, A.M. Gubens, C.B. Bastian, S.B. Taylor, C.J. Doyle and G.T. Bivona, Novel computational method for predicting polytherapy switching strategies to overcome tumor heterogeneity and evolution. Sci. Rep. 7 (2017) 1-14.

[45] G. Kalemkerian, W. Akerley, P. Bogner, H. Borghaei, L. Chow, R. Downey, L. Gandhi, A. Ganti, R. Govindan, et al., Non-small cell lung cancer. J. Natl. Comprehensive Cancer Netw. 10 (2012) 1236-1271. 
[46] N. Komarova, Stochastic modeling of drug resistance in cancer. Theor. Popul. Biol. 239 (2006) 351-366.

[47] M. Kimmel, A. Swierniaka and A. Polanski, Infinite-dimensional model of evolution of drug resistance of cancer cells. J. Math. Syst. Estim. Control 8 (1998) 1-16.

[48] O. Lavi, M.M. Gottesman and D. Levy, The dynamics of drug resistance: a mathematical perspective. Drug Resist. Updates 15 (2012) 90-97.

[49] T. Lorenzi, H.R. Chisholm and J. Clairambault, Tracking the evolution of cancer cell populations through the mathematical lens of phenotype-structured equations. Biol. Dir. 11 (2016) 1-17.

[50] T. Lorenzi, H.R. Chisholm, L. Desvillettes and D.B. Hughes, Dissecting the dynamics of epigenetic changes in phenotypestructured populations exposed to fluctuating environments. J. Theor. Biol. 386 (2015) 166-176.

[51] A. Lorz, T. Lorenzi, E.M. Hochberg, J. Clairambault and B. Perthame, Populational adaptive evolution, chemotherapeutic resistance and multiple anti-cancer therapies. ESAIM: M2AN 47 (2013) 377-399.

[52] A. Lorz, T. Lorenzi, J. Clairambault, A. Escargueil and B. Perthame, Modeling the effects of space structure and combination therapies on phenotypic heterogeneity and drug resistance in solid tumors. Bull. Math. Biol. 77 (2015) 1-22.

[53] C.C. Maley, A. Aktipis, A.T. Graham, A. Sottoriva, M.A. Boddy, M. Janiszewska, S.A. Silva, M. Gerlinger, Y. Yuan, J.K. Pienta, et al., Classifying the evolutionary and ecological features of neoplasms. Nat. Rev. Cancer 17 (2017) 605-619.

[54] A. Marusyk, V. Almendro and K. Polyak, Intra-tumour heterogeneity: a looking glass for cancer? Nat. Rev. Cancer 12 (2012) $323-334$.

[55] L. Merlo, J. Pepper, B. Reid and C. Maley, Cancer as an evolutionary and ecological process. Nat. Rev. Cancer 6 (2006) 924-935.

[56] K. Masui, B. Gini, J. Wykosky, C. Zanca, P.S. Mischel, F.B. Furnari and W.K. Cavenee, A tale of two approaches: Complementary mechanisms of cytotoxic and targeted therapy resistance may inform next-generation cancer treatments. Carcinogenesis 34 (2013) 725-738.

[57] J.P. Medema, Cancer stem cells: the challenges ahead. Nat. Cell Biol. 15 (2013) 338-344.

[58] F. Michor, A.M. Nowak and Y. Iwasa, Evolution of Resistance to Cancer Therapy. Curr. Pharm. Des. 12 (2006) $261-271$.

[59] S. Misale, I. Bozic, J. Tong, A. Peraza-Penton, A. Lallo, F. Baldi, K. Lin, M. Truini, L. Trusolino, A. Bertotti, F. Di Nicolantonio, M. Nowak, L. Zhang, K. Wood and A. Bardelli, Vertical suppression of the EGFR pathway prevents onset of resistance in colorectal cancers. Nat. Commun. 6 (2015) 1-9.

[60] S.T. Mok, Y.-L. Wu, C.-J. Yu, C. Zhou, Y.-M. Chen, L. Zhang, J. Ignacio, M. Liao, V. Srimuninnimit, et al., Randomized, placebo-controlled, phase II study of sequential Erlotinib and chemotherapy as first-line treatment for advanced non-small-cell lung cancer. J. Clin. Oncol. 27 (2009) 5080-5087.

[61] E. Moreno, Is cell competition relevant to cancer? Nat. Rev. Cancer 8 (2008) 141-147.

[62] E. Moreno, K. Basler and G. Morata, Cells compete for decapentaplegic survival factor to prevent apoptosis in Drosophila wing development. Nature 416 (2002) 755-759.

[63] M.S. Mumenthaler, J. Foo, C.N. Choi, N. Heise, K. Leder, B.D. Agus, W. Pao, F. Michor and P. Mallick, The impact of microenvironmental heterogeneity on the evolution of drug resistance in cancer cells. Cancer Inf. 14 (2015) 19-31.

[64] J. Murray, Mathematical Biology. Springer-Verlag (2002).

[65] B. Perthame and G. Barles, Dirac concentrations in Lotka-Volterra parabolic PDEs. Indiana Univ. Math. J. 57 (2008) 3275-3301.

[66] E. Piretto, M. Delitala and M. Ferraro, Combination therapies and intra-tumoral competition: Insights from mathematical modeling. J. Theor. Biol. 446 (2018) 149-159.

[67] O.A. Pisco, A. Brock, J. Zhou, A. Moor, M. Mojtahedi, D. Jackson and S. Huang, Non-darwinian dynamics in therapy-induced cancer drug resistance. Nat. Commun. 4 (2013) 2467.

[68] C. Pouchol and E. Trélat, Global stability with selection in integro-differential Lotka-Volterra systems modelling traitstructured populations. J. Biol. Dyn. 12 (2018) 872-893.

[69] C. Pouchol, J. Clairambault, A. Lorz and E. Trélat, Asymptotic analysis and optimal control of an integro-differential system modelling healthy and cancer cells exposed to chemotherapy. J. Math. Pures Appl. 116 (2018) 268-308.

[70] B. Perthame, F. Quirós and L.J. Vázquez, The Hele-Shaw asymptotics for mechanical models of tumor growth. Arch. Rational Mech. Anal. 212 (2014) 93-127.

[71] L. Peng, D. Trucu, P. Lin, A. Thompson and A.J.M. Chaplain, A multiscale mathematical model of tumour invasive growth. Bull. Math. Biol. 79 (2016) 389-429.

[72] J.M. Rowe and B. Löwenberg, Gemtuzumab ozogamicin in acute myeloid leukemia: a remarkable saga about an active drug. Blood 121 (2013) 4838-4841.

[73] T.J. Ribeiro, T.L. Macedo, G. Curigliano, L. Fumagalli, M. Locatelli, M. Dalton, A. Quintela, B.J. Carvalheira, S. Manunta, L. Mazzarella, J. Brollo and A. Goldhirsch, Cytotoxic drugs for patients with breast cancer in the era of targeted treatment: Back to the future? Ann. Oncol. 23 (2012) 547-555.

[74] T. Roose, S.J. Chapman and P.K. Maini, Mathematical models of avascular tumor growth. SIAM Rev. 49 (2007) $179-208$.

[75] V.S. Sharma, Y.D. Lee, B. Li, P.M. Quinlan, F. Takahashi, S. Maheswaran, U. McDermott, N. Azizian, L. Zou and M.A. Fischbach, A chromatin-mediated reversible drug-tolerant state in cancer cell subpopulations. Cell 141 (2010) 69-80.

[76] S. Singh, K.N. Tank, P. Dwiwedi, J. Charan, R. Kaur, P. Sidhu and K.V. Chugh, Monoclonal antibodies: a review. Curr. Clin. Pharmacol. 13 (2017) 85-99.

[77] P. Simpson, Parameters of cell competition in the compartments of the wing disc of Drosophila. Dev. Biol. 69 (1979) 182-193. 
[78] M. Slingerland, H. Guchelaar and H. Gelderblom, Liposomal drug formulations in cancer therapy: 15 years along the road. Drug Discov. Today 17 (2012) 160-166.

[79] S. Suijkerbuijk, G. Kolahgar, I. Kucinski and E. Piddini, Cell competition drives the growth of intestinal adenomas in Drosophila. Curr. Biol. 26 (2016) 428-438.

[80] A. Swierniak, M. Kimmel and J. Smieja, Mathematical modeling as a tool for planning anticancer therapy. Eur. J. Pharmacol. 625 (2009) 108-121.

[81] B.A. Teicher, Cancer Drug Resistance. Humana Press, Totowa, N.J. (2006).

[82] A. Tsuboi, S. Ohsawa, D. Umetsu, Y. Sando, E. Kuranaga, T. Igaki and K. Fujimoto, Competition for space is controlled by apoptosis-induced change of local epithelial topology. Curr. Biol. 28 (2018) 2115-2128.

[83] O. Trédan, M.C. Galmarini, K. Patel and I.F. Tannock, Drug resistance and the solid tumor microenvironment. J. Natl. Cancer Inst. 99 (2007) 1441-1454.

[84] S. Vivarelli, L. Wagstaff and E. Piddini, Cell wars: regulation of cell survival and proliferation by cell competition. Essays Biochem. 53 (2012) 69-82.

[85] L. Wagstaff, G. Kolahgar and E. Piddini, Competitive cell interactions in cancer: a cellular tug of war. Trends Cell Biol. 23 (2013) 160-167.

[86] K. Wosikowski, A.J. Silverman, P. Bishop, J. Mendelsohn and E.S. Bates, Reduced growth rate accompanied by aberrant epidermal growth factor signaling in drug resistant human breast cancer cells. Biochim. Biophys. Acta 1497 (2000) $215-226$.

[87] N. Yoon, R. Velde, A. Marusyk and J. Scott, Optimal therapy scheduling based on a pair of collaterally sensitive drugs. Bull. Math. Biol. (2018) 1-34.

[88] Z. Zhang, C.J. Lee, L. Lin, V. Olivas, V. Au, M. Abdel-rahman, X. Wang, D.A. Levine, J. Kyung, J.Y. Choi, C.-M. Choi, S.-W. Kim, J.S. Jang, S.Y. Park, S.W. Kim, H.D. Lee, J.-S. Lee, V. Miller and M. Arcila, Activation of the AXL kinase causes resistance to EGFR-targeted therapy in lung cancer. Nat. Genet. 44 (2012) 852-860. 